\title{
Hybrid Halide Perovskites: Fundamental Theory and Materials Design
}

\author{
Marina R. Filip ${ }^{1}$ and George Volonakis ${ }^{1}$ \\ ${ }^{1}$ Department of Materials, \\ University of Oxford, \\ Parks Road, Oxford OX1 3PH, \\ United Kingdom \\ Feliciano Giustino ${ }^{1,2}$ ? \\ ${ }^{1}$ Department of Materials, \\ University of Oxford, \\ Parks Road, Oxford OX1 3PH, \\ United Kingdom \\ ${ }^{2}$ Department of Materials Science and Engineering, \\ Cornell University, \\ Ithaca, New York 14853, \\ USA
}

\begin{abstract}
Hybrid organic-inorganic halide perovskites have emerged as a disruptive new class of materials, exhibiting optimum properties for a broad range of optoelectronic applications, most notably for photovoltaics. The first report of highly efficient organicinorganic perovskite solar cells in 2012 (Lee et al. 2012 ) marked a new era for photovoltaics research, reporting a power conversion efficiency of over 10\% (NREL 2017). Only five years after this discovery, perovskite photovoltaic devices have reached a certified efficiency of $22.7 \%$, making them the first solution processable technology to surpass thin film and multi-crystalline silicon solar cells (NREL 2017). The remarkable development of perovskite solar cells is due to the ideal optoelectronic properties of organicinorganic lead-halide perovskites. The prototypical compound, methylammonium lead iodide, $\mathrm{CH}_{3} \mathrm{NH}_{3} \mathrm{PbI}_{3}$ (Stranks and Snaith 2015) is a direct band gap semiconductor with a band gap in the visible, high charge carrier mobility, long diffusion length and low excitonic binding energy (Johnston and Herz 2016). Due to these ideal properties, $\mathrm{CH}_{3} \mathrm{NH}_{3} \mathrm{PbI}_{3}$ is also drawing interest across many other applications beyond photovoltaics, such as light emitting devices (Tan et al. 2014), lasers (Wehrenfennig et al. 2014), photocatalysts (Chen et al. 2015) and transistors (Ward et al. 2017). The continued progress of metal-halide perovskite optoelectronics relies not only on a detailed understanding of the electronic and optical properties of materials in this class, but also on the development of practical strategies to tune their properties by controlling parameters such as chemical composition. In this context, $a b$ initio computational modelling can play a key role in providing a physical interpretation of experimental measurements, and guiding the design of novel halide perovskites with tailored properties. In this chapter we will present an account of the contributions to this fast developing field of research from our computational modelling group. The chapter is organized in two sections. The first section focuses on the structural and optoelectronic properties of $\mathrm{CH}_{3} \mathrm{NH}_{3} \mathrm{PbI}_{3}$. Here, we expand on some of the challenging aspects of modelling the electronic and vibrational properties of $\mathrm{CH}_{3} \mathrm{NH}_{3} \mathrm{PbI}_{3}$, and discuss the main theoretical results alongside experimental data. The second section discusses the recent computationally-led materials design of novel halide perovskites, and the principal challenges in replacing $\mathrm{Pb}^{2+}$ in $\mathrm{CH}_{3} \mathrm{NH}_{3} \mathrm{PbI}_{3}$ by non-toxic elements.
\end{abstract}

\section{METHYLAMMONIUM LEAD IODIDE}

Methylammonium lead-iodide, $\mathrm{CH}_{3} \mathrm{NH}_{3} \mathrm{PbI}_{3}$, belongs to the $\mathrm{ABX}_{3}$ perovskite structural family (Poglitsch and Weber, 1987). As shown in FIG. 1, the $\mathrm{Pb}^{2+}$ and $\mathrm{I}^{-}$ ions form a three-dimensional network of corner-sharing octahedra. The organic $\mathrm{CH}_{3} \mathrm{NH}_{3}^{+}$cations occupy the center of the cuboctahedral cavities enclosed by the inorganic $\mathrm{PbI}_{6}$ network (Poglitsch and Weber, 1987).

* feliciano.giustino@materials.ox.ac.uk
The crystal structure of $\mathrm{CH}_{3} \mathrm{NH}_{3} \mathrm{PbI}_{3}$ is strongly dependent on temperature and undergoes two phase transitions (Baikie et al., 2013, Poglitsch and Weber, 1987, Stoumpos et al. | 2013||Weller et al.| 2015): from the low temperature orthorhombic Pnma structure to the tetragonal $I 4 / \mathrm{mcm}$ structure at $160 \mathrm{~K}$, and the cubic $P m \overline{3} m$ structure at 315-330 K. As shown in FIG. 1, all phases of $\mathrm{CH}_{3} \mathrm{NH}_{3} \mathrm{PbI}_{3}$ maintain the corner sharing connectivity of the $\mathrm{PbI}_{6}$ octahedra, however the degree of octahedral tilting is reduced as the temperature increases. In addition, the organic $\mathrm{CH}_{3} \mathrm{NH}_{3}^{+}$cations exhibit ordered orientations throughout the unit cell only in the orthorhombic phase, while in the tetragonal and cubic phase the cations are 
a

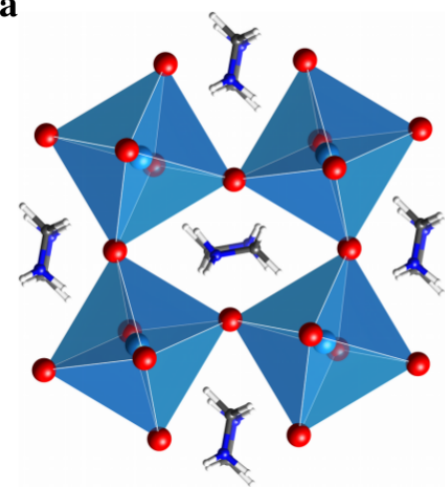

b

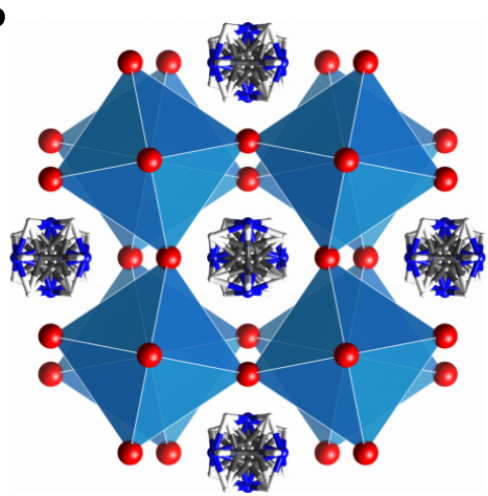

c

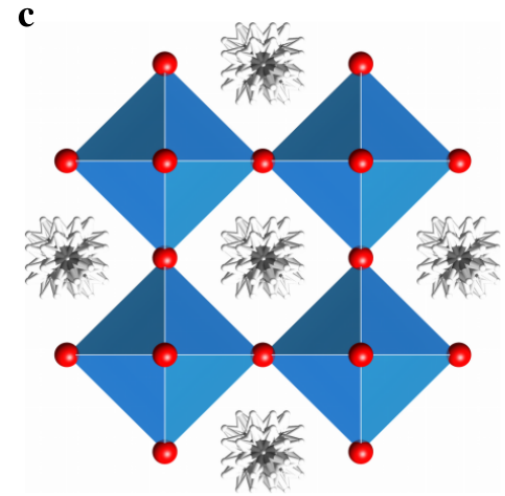

FIG. 1 Temperature dependent crystal structure of $\mathbf{C H}_{3} \mathbf{N H}_{3} \mathbf{P b I}_{3}$. Polyhedral representation of the low temperature orthorhombic Pnma structure (a), the tetragonal $I 4 / \mathrm{mcm}$ structure (b) and the cubic Pm $\overline{3} m$ structure (c). In all three panels the $\mathrm{Pb}$ atoms are represented by the light blue spheres at the center of the octahedra, the I atoms are the red spheres at the corners of the octahedra, and the $\mathrm{C}, \mathrm{N}$ and $\mathrm{H}$ atoms are represented by the dark grey, dark blue, and white spheres, respectively. The figures were constructed using the lattice parameters and atomic positions reported by (Weller et al., 2015).

orientationally disordered (Baikie et al., 2013, Poglitsch and Weber, 1987, Stoumpos et al., 2013; Weller et al. 2015).

The excited state properties of $\mathrm{CH}_{3} \mathrm{NH}_{3} \mathrm{PbI}_{3}$ have been intensely investigated over the last five years. The optical absorption spectrum of $\mathrm{CH}_{3} \mathrm{NH}_{3} \mathrm{PbI}_{3}$ is characteristic of a direct band gap semiconductor (Herz, 2016), with a sharp absorption onset around $1.6 \mathrm{eV}$. At low temperature, the optical absorption spectrum exhibits an excitonic peak, with an exciton binding energy of $\sim 20 \mathrm{meV}$. This feature becomes less visible as the temperature increases (D'Innocenzo et al., 2014, Herz, 2016. Miyata et al., 2015). In addition, the absorption lineshape at room temperature exhibits an Urbach tail of $15 \mathrm{meV}$ (De Wolf et al., 2014), unexpectedly low given the disordered character of the $\mathrm{CH}_{3} \mathrm{NH}_{3}^{+}$cation. The optical band gap of $\mathrm{CH}_{3} \mathrm{NH}_{3} \mathrm{PbI}_{3}$ increases with temperature from $1.62 \mathrm{eV}$ at $4 \mathrm{~K}$ to $1.67 \mathrm{eV}$ at $160 \mathrm{~K}$. At the phase transition temperature between the orthorhombic and tetragonal phase, $160 \mathrm{~K}$, the band gap decreases sharply to approximately $1.58 \mathrm{eV}$, and then continues to increase beyond this temperature, as in the orthorhombic phase (Milot et al. 2015).

Free electrons and holes dominate over bound exciton pairs upon photoexcitation, an ideal characteristic for application in solar energy conversion (D'Innocenzo et al. 2014, Herz, 2016). The photogenerated charge carriers are long lived in $\mathrm{CH}_{3} \mathrm{NH}_{3} \mathrm{PbI}_{3}$ and exhibit charge carrier mobilities of approximately $35 \mathrm{~cm}^{2}(V s)^{-1}$ (Johnston and Herz, 2016) at room temperature. Bimolecular recombination in $\mathrm{CH}_{3} \mathrm{NH}_{3} \mathrm{PbI}_{3}$ is found to be the predominant mechanism for charge carrier recombination at standard photovoltaic device operation conditions, exhibiting a highly non-Langevin behavior (Herz, 2016, Johnston and Herz, 2016). This means that the ratio between the bimolecular recombination rate $k_{2} \sim 10^{-10} \mathrm{~cm}^{3} \mathrm{~s}^{-1}$ and the charge carrier mobility $\mu \sim 10 \mathrm{~cm}^{2} \mathrm{~V}^{-1} \mathrm{~s}^{-1}$ is four orders of magnitude lower than the value $e /\left(\epsilon_{0} \epsilon_{r}\right)$ predicted by the Langevin model (Herz, 2016, Wehrenfennig et al. 2014). This effect explains the long charge carrier diffusion lengths $(\sim 1 \mu \mathrm{m})$ measured for halide perovskites (Herz, 2016).

The band gap of $\mathrm{CH}_{3} \mathrm{NH}_{3} \mathrm{PbI}_{3}$ can be tuned via chemical substitution (Herz, 2016). The replacement of the $\mathrm{CH}_{3} \mathrm{NH}_{3}^{+}$cation with $\mathrm{CH}\left(\mathrm{NH}_{2}\right)_{2}^{+}$(formamidinium), $\mathrm{Cs}^{+}$ or $\mathrm{Rb}^{+}$leads to a change in the optical band gap over a range of $0.3 \mathrm{eV}$ (Eperon et al., 2014; Filip et al., 2014); the substitution of $\mathrm{Pb}^{2+}$ by $\mathrm{Sn}^{2+}$ reduces the band gap by approximately $0.4 \mathrm{eV}$ (Hao et al. 2014 ) and the replacement of $\mathrm{I}^{-}$by the smaller anions $\mathrm{Cl}^{-}$and $\mathrm{Br}^{-}$blue-shifts the optical band gap by up to $0.7 \mathrm{eV}$ (Comin et al. 2015 . Noh et al. 2013). In practice, mixed cation, mixed metal and mixed halide perovskites are currently explored in order to optimize light absorption over the entire visible spectrum, but also to improve the stability of perovskite solar cells (Eperon et al., 2016, McMeekin et al., 2016, Saliba et al. |2016).

Theoretical investigations of the fundamental structural, electronic and vibrational properties of $\mathrm{CH}_{3} \mathrm{NH}_{3} \mathrm{PbI}_{3}$ have played an important role in the interpretation of the experimental data summarized above. In the following we discuss some of the theoretical results obtained by us and our coworkers in the study of $\mathrm{CH}_{3} \mathrm{NH}_{3} \mathrm{PbI}_{3}$.

\section{A. Basic Electronic Structure of $\mathbf{C H}_{3} \mathbf{N H}_{3} \mathbf{P b l}_{3}$}

To construct the electronic band structure of $\mathrm{CH}_{3} \mathrm{NH}_{3} \mathrm{PbI}_{3}$ it is necessary to know the lattice parameters and the atomic positions for the perovskite unit cell. For the low-temperature orthorhombic phase, the unit cell was fully resolved for the first time by (Baikie et al. , 2013). The unit cell contains four $\mathrm{PbI}_{6}$ octahedra and four $\mathrm{CH}_{3} \mathrm{NH}_{3}^{+}$cations, 48 atoms in total, 


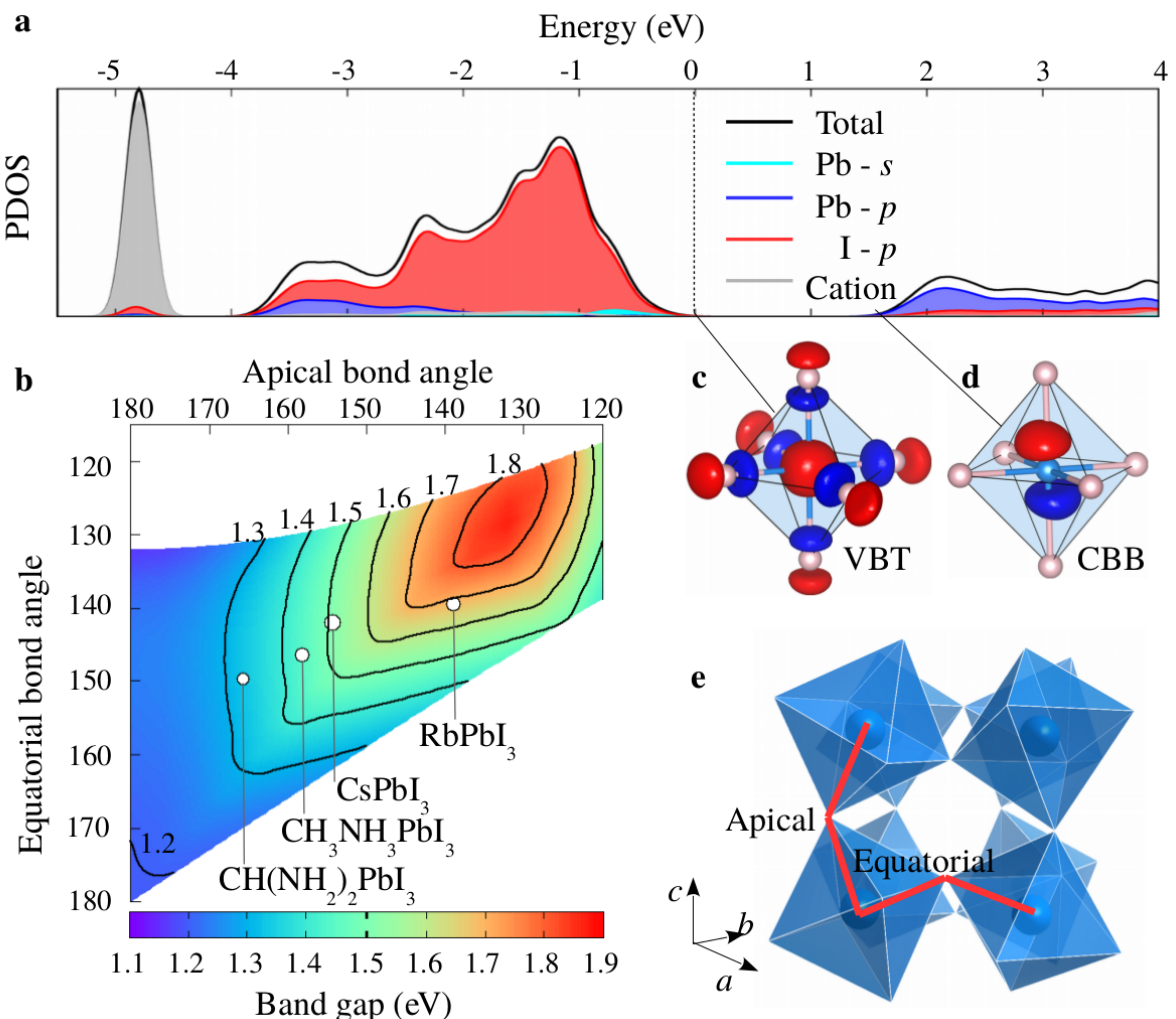

FIG. 2 Electronic structure and band gap tunability of $\mathbf{C H}_{3} \mathbf{N H}_{3} \mathbf{P b I}_{3}$. a. Projected density of states of $\mathrm{CH}_{3} \mathrm{NH}_{3} \mathrm{PbI}_{3}$ calculated within the local density approximation to density functional theory (DFT/LDA) without spin-orbit coupling for the orthorhombic crystal structure. The bands are aligned to the valence band top (VBT) represented by the dotted line. Adapted with permission from (Filip et al. 2014), Copyright (2014) Nature Publishing Group. b. Two-dimensional map of the DFT band gap as a function of the apical and equatorial bond angles. The band gaps for each point of the map were calculated for Platonic model structures, as described by (Filip et al. 2014 ), that do not contain the A-site cation, as shown in e. The white disks represent the angular coordinates (apical and equatorial bond angles) of realistic models of $\mathrm{CH}_{3} \mathrm{NH}_{3} \mathrm{PbI}_{3}, \mathrm{CH}_{\left(\mathrm{NH}_{2}\right)} \mathrm{PbI}_{3}$, $\mathrm{CsPbI}_{3}$ and $\mathrm{RbPbI}_{3}$. Adapted with permission from (Filip et al. 2014 ), Copyright (2014) Nature Publishing Group. c - d. Wave functions corresponding to the valence band top (c) and the conduction band bottom of a lead-iodide perovskite (d). e. Polyhedral representation of the Platonic model for the orthorhombic perovskite structure. The apical and equatorial bond angles are represented by the red lines in the figure.

arranged according to the Pnma space group.

In the tetragonal and cubic phases, the unit cell contains four and one $\mathrm{PbI}_{6}$ octahedra, respectively, and the shape of the inorganic octahedral networks are well described (Stoumpos et al., 2013: Weller et al., 2015) by the space groups $I 4 / \mathrm{mcm}$ and $P m 3 m$, respectively. At variance with the inorganic scaffold, the organic cations have a disordered orientation inside the perovskite cavity, in the tetragonal and cubic phases, and pose difficulties for any electronic structure calculation that employs periodic boundary conditions. For example, structural relaxation of the unit cell starting from arbitrary orientations of the $\mathrm{CH}_{3} \mathrm{NH}_{3}^{+}$cations leads to significant distortions of the unit cell and considerable changes to the electronic band structure that are highly dependent on the choice of the orientation (Motta et al. 2015). This effect is purely an artefact of the chosen orientation of the cation, and bears no physical meaning.
To ensure that we can obtain meaningful results of the electronic properties of $\mathrm{CH}_{3} \mathrm{NH}_{3} \mathrm{PbI}_{3}$ we have two options. One approach is to simulate the disordered orientation of the $\mathrm{CH}_{3} \mathrm{NH}_{3}^{+}$by averaging over its many possible orientations. This approach is best addressed using molecular dynamics (Carignano and Kachmar, 2015 , Lahnsteiner et al. 2016) and requires a careful study of the size of the supercell in order to correctly represent the disordered orientations of $\mathrm{CH}_{3} \mathrm{NH}_{3}^{+}$. The second approach relies on the general property of $\mathrm{ABX}_{3}$ perovskites by which the A-site cations do not participate in bonds, and as a consequence they only contribute to electronic states far away from the band edges (Megaw, 1973). In this latter approach, we can first calculate the electronic properties of $\mathrm{CH}_{3} \mathrm{NH}_{3} \mathrm{PbI}_{3}$ in the well known orthorhombic phase. Then we can investigate how the change in the shape of the inorganic octahedral network affects these properties. In the following, we describe the latter strat- 
egy.

In FIG. 2 a we show the projected density of states of $\mathrm{CH}_{3} \mathrm{NH}_{3} \mathrm{PbI}_{3}$ in the low temperature orthorhombic Pnma case. The valence band top is occupied by I- $p$ and $\mathrm{Pb}-s$ electrons, while the conduction band bottom is of I- $p$ and $\mathrm{Pb}-p$ character. As expected, the states localized on the $\mathrm{CH}_{3} \mathrm{NH}_{3}^{+}$cation are located away from the band edges (approximately $5 \mathrm{eV}$ ). Based on FIG. 2 a we can construct a tight-binding argument to explain the tunability of the band gap by substitution of I anions or $\mathrm{Pb}$ cations. The replacement of $\mathrm{I}$ by $\mathrm{Cl}$ or $\mathrm{Br}$ leads to an increase in the band gap, due to the lower-lying Br- $p$ and Cl- $p$ electronic states that populate the valence band top; similarly, the substitution of $\mathrm{Pb}$ by $\mathrm{Sn}$ lowers the conduction band bottom and reduces the band gap due to the lower-lying Sn- $p$ states. In addition, from FIG. 1a, it is clear that the $\mathrm{CH}_{3} \mathrm{NH}_{3}^{+}$cations do not contribute to the optical absorption, and their role is to act as a structural filler and to ensure the charge neutrality of the unit cell. In practice, the $\mathrm{CH}_{3} \mathrm{NH}_{3}^{+}$cation can be replaced by a positive background charge, and the electronic band structure would not change significantly (Filip et al., 2014).

Taking advantage of this property, we can study how the shape of the cuboctahedral cavity influences the electronic structure of the perovskite. (Filip et al., 2014) showed that the unit cell of the perovskite lattice can be uniquely described by a 'Platonic' model, whereby the $\mathrm{PbI}_{6}$ octahedra are ideal and identical throughout the lattice, and their orientation within the unit cell is uniquely described by two angles: the equatorial and the apical bond angles (FIG. 2p). FIG. 2p depicts the variation of the band gap with the two bond angles of the 'Platonic' perovskite lattice. The gap increases as the bond angles decrease.

Two observations can be drawn from FIG. 2b. Firstly, the size of the A-site cation in the perovskite lattice influences the shape of the octahedral lattice, by changing the apical and equatorial bond angles, which in turn enables the control of the band gap. This leads to the following simple trend: the smaller the cation, the smaller the bond angles, the larger the band gap. The band gap trend predicted in FIG. $2 \mathrm{~b}$ is in agreement with the trend observed experimentally, and explains the band gap tunability with the substitution of the A-site cation (Eperon et al., 2014; Filip et al. 2014). Secondly, based on FIG. 2 b we can explain the discontinuity in the band gap dependence on temperature observed at the transition temperature of $160 \mathrm{~K}$ (Milot et al., 2015). When the $\mathrm{CH}_{3} \mathrm{NH}_{3} \mathrm{PbI}_{3}$ transitions from an orthorhombic to a tetragonal phase, the apical bond angle increases from $160^{\circ}$ to $180^{\circ}$. According to the map in FIG.2p, the band gap should decrease by up to $0.2 \mathrm{eV}$, in good agreement with the sudden drop of $0.1 \mathrm{eV}$ measured around $160 \mathrm{~K}$. The core conclusion that can be drawn from FIG. 2a is that the fundamental roles of the organic cation $\mathrm{CH}_{3} \mathrm{NH}_{3}^{+}$ in the lead-iodide perovskite lattice are to balance the charge, and to stabilize the shape of the perovskite lattice. Therefore, in order to ensure that we are studying a physically meaningful crystal structure, in the following discussions we focus on the low-temperature orthorhombic Pnma phase, as refined by (Baikie et al., 2013).

\section{B. The Quasiparticle Band Structure of $\mathrm{CH}_{3} \mathbf{N H}_{3} \mathbf{P b l}_{3}$}

The calculations shown so far in FIG. 2 were obtained within DFT in the scalar-relativistic approximation. However, the presence of $\mathrm{Pb}$ in $\mathrm{CH}_{3} \mathrm{NH}_{3} \mathrm{PbI}_{3}$ requires a relativistic treatment. In FIG. 3 a we show that the spinorbit coupling effect is crucial for the correct calculation of the band edge topology. Not only relativistic effects lower the conduction band bottom of $\mathrm{CH}_{3} \mathrm{NH}_{3} \mathrm{PbI}_{3}$ by approximately $1 \mathrm{eV}$ (Even et al., 2013), but also they change the shape of the band edges. In particular, the effective masses become fully isotropic upon inclusion of spin-orbit coupling, by contrast with those calculated in the scalar-relativistic approach (Menéndez-Proupin et al. 2014). As shown in Table I, fully-relativistic DFT calculations underestimate the band gap by $\sim 1 \mathrm{eV}$ and the reduced effective mass by a factor of two.

The underestimation of the band gap and effective masses of DFT can be corrected by including quasiparticle effects. The quasiparticle band gap and band structure can be calculated within the $G W$ approximation (Hedin, 1965). The quasiparticle eigenvalues $E_{n \mathbf{k}}$ are calculated starting from the single-particle DFT/LDA eigenvalues $\epsilon_{n \mathbf{k}}$ as (Hedin, 1965; Hybertsen and Louie, 1986):

$$
E_{n \mathbf{k}}=\epsilon_{n \mathbf{k}}+Z\left(\epsilon_{n \mathbf{k}}\right)\left\langle n \mathbf{k}\left|\hat{\Sigma}\left(\epsilon_{n \mathbf{k}}\right)-V_{x c}\right| n \mathbf{k}\right\rangle,
$$

where $|n \mathbf{k}\rangle$ denotes a single-particle eigenstate with band index $n$ and crystal momentum $\mathbf{k}, Z(\omega)=(1-$ $\partial \operatorname{Re} \hat{\Sigma} / \partial \omega)^{-1}$ is the quasiparticle renormalization, $V_{x c}$ is the exchange-correlation potential, and $\hat{\Sigma}=i G_{0} W_{0}$ is the quasiparticle self-energy (Hybertsen and Louie, 1986). In the expression of $\hat{\Sigma}, G_{0}$ is the non-interacting Green's function calculated using single-particle eigenvalues and eigenstates as obtained from DFT. The screened Coulomb interaction has the expression $W_{0}=\epsilon^{-1} v$, where $v$ is the bare Coulomb interaction and $\epsilon^{-1}$ is the inverse dielectric matrix.

In the case of $\mathrm{CH}_{3} \mathrm{NH}_{3} \mathrm{PbI}_{3}$ it has been shown that the one-shot $G_{0} W_{0}$ quasiparticle band gap is underestimated with respect to experiment by up to $0.4 \mathrm{eV}$ (Brivio et al. 2014 Filip and Giustino, 2014), as show in Table I. This is primarily due to the underestimated single particle band gap calculated at the DFT level, which leads to an overestimation of the dielectric screening in $W_{0}$ (Brivio et al. 2014, Filip and Giustino, 2014). The quasiparticle band gap underestimation at the $G_{0} W_{0}$ level appears irrespective of the exchange-correlation functional employed for the DFT starting point, and it tends to worsen 

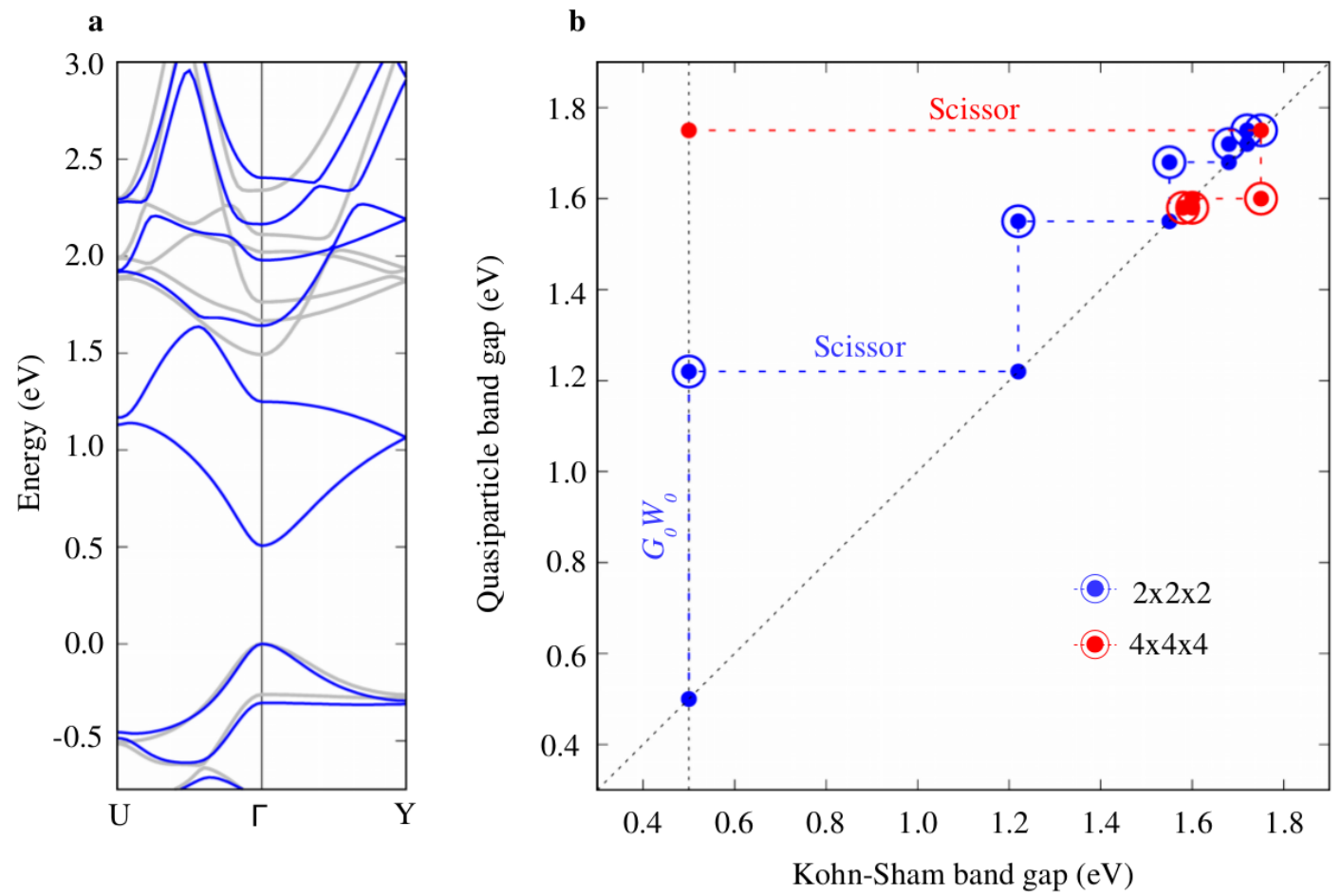

FIG. 3 Band structure of $\mathbf{C H}_{3} \mathbf{N H}_{3} \mathbf{P b I}_{3}$. a. DFT/LDA band structure of $\mathrm{CH}_{3} \mathrm{NH}_{3} \mathrm{PbI}_{3}$ calculated with (blue line) and without (grey line) including spin-orbit coupling effects. The calculations were performed for the orthorhombic Pnma phase using norm-conserving Troullier Martins pseudopotentials as described by (Filip and Giustino, 2014). b. Convergence of the self-consistent scissor $G W$ scheme for the quasiparticle band gap of $\mathrm{CH}_{3} \mathrm{NH}_{3} \mathrm{PbI}_{3}$. The self-consistent scissor $G W$ scheme is described in detail by (Filip and Giustino, 2014). For all quasiparticle calculations we have used 1000 bands, and plane wave cutoffs of 35 Ry and 6 Ry for the exchange and polarizability respectively. The diagram shows the self-consistent scissor scheme calculated for a $\Gamma$-centered $2 \times 2 \times 2 \mathbf{k}$-point grid (blue) and a $4 \times 4 \times 4 \mathbf{k}$-point grid (red). All quasiparticle calculations include semicore $d$ electrons for both $\mathrm{Pb}$ and I, as discussed by (Filip and Giustino, 2014).

when more core electrons are included in the valence configuration of I (Scherpelz et al. 2016$)$. However, this sensitivity to the DFT starting point can be mitigated by calculating the electron self-energy self-consistently (van Schilfegaarde et al., 2006).

FIG. $3 \mathrm{~b}$ shows an approximate self-consistency scheme introduced by (Kioupakis et al., 2008) and applied to the case of $\mathrm{CH}_{3} \mathrm{NH}_{3} \mathrm{PbI}_{3}$ by (Filip and Giustino, 2014), the self-consistent scissor $G W$. In this approach the $G_{0} W_{0}$ band gap correction $\Delta$ is first calculated, and then a scissor correction of magnitude $\Delta$ is applied to all eigenstates in the conduction band. These two steps are repeated until the band gap is converged, as shown in FIG. $3 \mathrm{~b}$ (Filip and Giustino, 2014, Kioupakis et al., 2008). The final converged band gap is of $1.57 \mathrm{eV}$, within $80 \mathrm{meV}$ of the measured optical band gap of $1.65 \mathrm{eV}$ (Davies et al., 2017; Milot et al., 2015). This small difference is accounted for by the absence of electron-hole interaction effects, and electron-phonon effects.

FIG. 4a shows the comparison between the $G W$ quasiparticle band structure and the DFT/LDA band structure obtained from Wannier interpolation (Marzari and Vanderbilt, 1997, Souza et al., 2001). Qualitatively the band dispersions calculated within DFT/LDA and $G W$ are similar, with the quasiparticle valence band exhibiting a noticeably more pronounced curvature. A more quantitative comparison between the DFT/LDA and $G W$ band dispersions is given by the isotropic effective masses, shown in Table I] The effective mass tensor is calculated as the inverse of the second derivatives of the energy with respect to the crystal momentum, $m_{i j}^{*}=\hbar^{2}\left(\partial^{2} \epsilon / \partial k_{i} \partial k_{j}\right)^{-1}$, and the values reported in Table I are the averages of the eigenvalues of the effective mass tensors. From Table \ we can see that the DFT/LDA and $G W$ effective masses differ by almost a factor of two. In addition, the $G W$ reduced effective mass of $0.11 m_{e}$ is in excellent agreement with the experimental value of $0.10 m_{e}$ reported by (Miyata et al. 2015 ), thus establishing the accuracy of the quasiparticle band structure calculations.

An important consequence of the quasiparticle effects in the calculation of the band structure of $\mathrm{CH}_{3} \mathrm{NH}_{3} \mathrm{PbI}_{3}$ is the band edge parabolicity. While both DFT/LDA and $G W$ yield isotropic band edges, the DFT/LDA bands are parabolic only in a narrow energy range near the band edges. On the other hand, the quasiparticle valence and 


\begin{tabular}{lccll}
\hline \hline & DFT & $G_{0} W_{0}$ & $G W$ & Exp. \\
\hline Band gap $(\mathrm{eV})$ & $0.50^{a, b, c}$ & $1.20^{a, b, c}$ & $1.57^{c}$ & $1.65^{c, d}$ \\
\hline$\epsilon_{\infty}$ & & $5.8^{b, e}$ & $6.5^{f}$ \\
$\epsilon_{0}$ & & $25.3^{e}$ & $30.5^{g}$ \\
\hline Hole effective mass $\left(m_{\mathrm{e}}\right)$ & $0.14^{b, c}$ & $0.18^{b, c}$ & $0.23^{b, c}$ & $\mathrm{~N} / \mathrm{A}$ \\
Electron effective mass $\left(m_{\mathrm{e}}\right)$ & $0.12^{b, c}$ & $0.16^{b, c}$ & $0.22^{b, c}$ & $\mathrm{~N} / \mathrm{A}$ \\
Reduced effective mass $\left(m_{\mathrm{e}}\right)$ & $0.06^{b, c}$ & $0.09^{b, c}$ & $0.11^{b, c}$ & $0.104 \pm 0.003^{h}$ \\
\hline Exciton binding energy $(\mathrm{meV})$ & & 44 & & $16^{h}$ \\
\hline \hline
\end{tabular}

TABLE I Calculated values of the band gap, high and low-frequency dielectric constants, electron, hole and reduced effective masses within DFT/LDA and within the $G W$ approximation including the self-consistent scissor correction of (Filip and Giustino, 2014). The band gaps are expressed in $\mathrm{eV}$ and the effective masses are expressed in the unit of electron masses $\left(m_{e}\right)$. The reduced effective mass is calculated using the expression: $\mu^{*}=\frac{m_{\mathrm{h}}^{*} m_{\mathrm{e}}^{*}}{m_{\mathrm{h}}^{*}+m_{\mathrm{e}}^{*}}$, where $m_{\mathrm{e}}^{*}, m_{\mathrm{h}}^{*}$ and $\mu^{*}$ are the electron, hole and reduced effective masses respectively. The exciton binding energy is calculated using the expression $E_{\mathrm{b}}=\mu^{*} / \epsilon_{\infty}^{2} \times \mathrm{E}_{\mathrm{Ry}}$ eV, with $E_{\mathrm{Ry}}=13.60565 \mathrm{eV}$, the reduced effective mass $\mu^{*}=0.11 m_{\mathrm{e}}$ and the high-frequency dielectric constant $\epsilon_{\infty}=5.8$.

${ }^{a}$ (Filip and Giustino, 2014)

${ }^{b}$ (Filip et al., 2015$)$

${ }^{c}$ (Davies et al. , 2017)

${ }^{d}$ (Milot et al., 2015$)$

e (Pérez-Osorio et al. 2015)

$f$ ("Hirasawa et al. 1994$)$

$g$ (Poglitsch and Weber, 1987)

$h$ (Miyata et al. 2015)

conduction band edges exhibit a parabolic profile over a range of $300 \mathrm{meV}$ (FIG. 4 $\mathrm{b}$ ). This observation is most clearly visualized in the joint density of states (JDOS), shown in FIG $4 \mathrm{~b}$, where the parabolic shape of the bands extends over approximately $0.3 \mathrm{eV}$ for the quasiparticle JDOS, while the JDOS calculated from DFT/LDA departs from the parabolic shape within $50 \mathrm{meV}$ from the onset.

The parabolicity of the conduction and valence band edges has important implications in the understanding of both absorption and recombination mechanisms in $\mathrm{CH}_{3} \mathrm{NH}_{3} \mathrm{PbI}_{3}$. Using a combined experimental and theoretical analysis of the optical absorption lineshape, (Davies et al. 2017) showed that the optical absorption spectrum of $\mathrm{CH}_{3} \mathrm{NH}_{3} \mathrm{PbI}_{3}$ can be modelled using the Elliot's theory (Elliott, 1957) up to $100 \mathrm{meV}$ above the absorption onset. Within this premise, (Davies et al., 2017) decoupled the excitonic and continuum part of the absorption spectrum, and calculated the bimolecular recombination rate using the van Roosbroek-Shockley formalism (van Roosbroek and Shockley, 1954). By comparing this model to direct transient spectroscopic measurements, (Davies et al., 2017) demonstrated that bimolecular recombination in $\mathrm{CH}_{3} \mathrm{NH}_{3} \mathrm{PbI}_{3}$, is an inverse absorption process. This result reinforces the conclusion that $\mathrm{CH}_{3} \mathrm{NH}_{3} \mathrm{PbI}_{3}$ is in many respects very similar to conventional semiconductors, such as GaAs (Herz, 2016).

\section{Vibrational Properties of $\mathrm{CH}_{3} \mathrm{NH}_{3} \mathrm{Pbl}_{3}$}

The vibrational spectrum is a key piece of information in the study of the optoelectronic properties of $\mathrm{CH}_{3} \mathrm{NH}_{3} \mathrm{PbI}_{3}$. Lattice vibrations can be analyzed experimentally from IR (Pérez-Osorio et al. 2015$)$ and Raman (Ledinský et al., 2015) spectroscopy, as well as inelastic neutron scattering (Družbicki et al., 2016), and can be directly compared with $a b$ initio calculations. Compared to the latter two techniques, IR spectroscopy is most accessible, as high-resolution measurements can be achieved in a short time frame over a wide range of frequencies (Pérez-Osorio et al. 2015$)$.

The vibrational properties of $\mathrm{CH}_{3} \mathrm{NH}_{3} \mathrm{PbI}_{3}$ can be calculated within density functional perturbation theory (DFPT). The vibrational spectrum of $\mathrm{CH}_{3} \mathrm{NH}_{3} \mathrm{PbI}_{3}$ in the orthorhombic Pnma phase consists of 144 normal modes at the $\Gamma$ point, with frequencies extending up to $3100 \mathrm{~cm}^{-1}$ (Pérez-Osorio et al. 2015). The vibrational modes of $\mathrm{CH}_{3} \mathrm{NH}_{3} \mathrm{PbI}_{3}$ can be classified into three main categories: vibrations of the $\mathrm{CH}_{3} \mathrm{NH}_{3}^{+}$cations, vibrations of the inorganic $\mathrm{PbI}_{3}$ network, and mixed modes. In addition, the vibrations of the $\mathrm{CH}_{3} \mathrm{NH}_{3}^{+}$cations can be decomposed into rigid translations, spinning vibrations around the $\mathrm{C}-\mathrm{N}$ axis, librations around the center of mass of the cation, and internal vibrations. Up to $60 \mathrm{~cm}^{-1}$, the main contribution to the vibrational phonon modes comes from the internal vibrations of the inorganic network, while above $60 \mathrm{~cm}^{-1}$ the vibrational phonon modes are almost exclusively due to the vibrations of the organic cations (Pérez-Osorio et al. 2015$)$. (Pérez-Osorio 


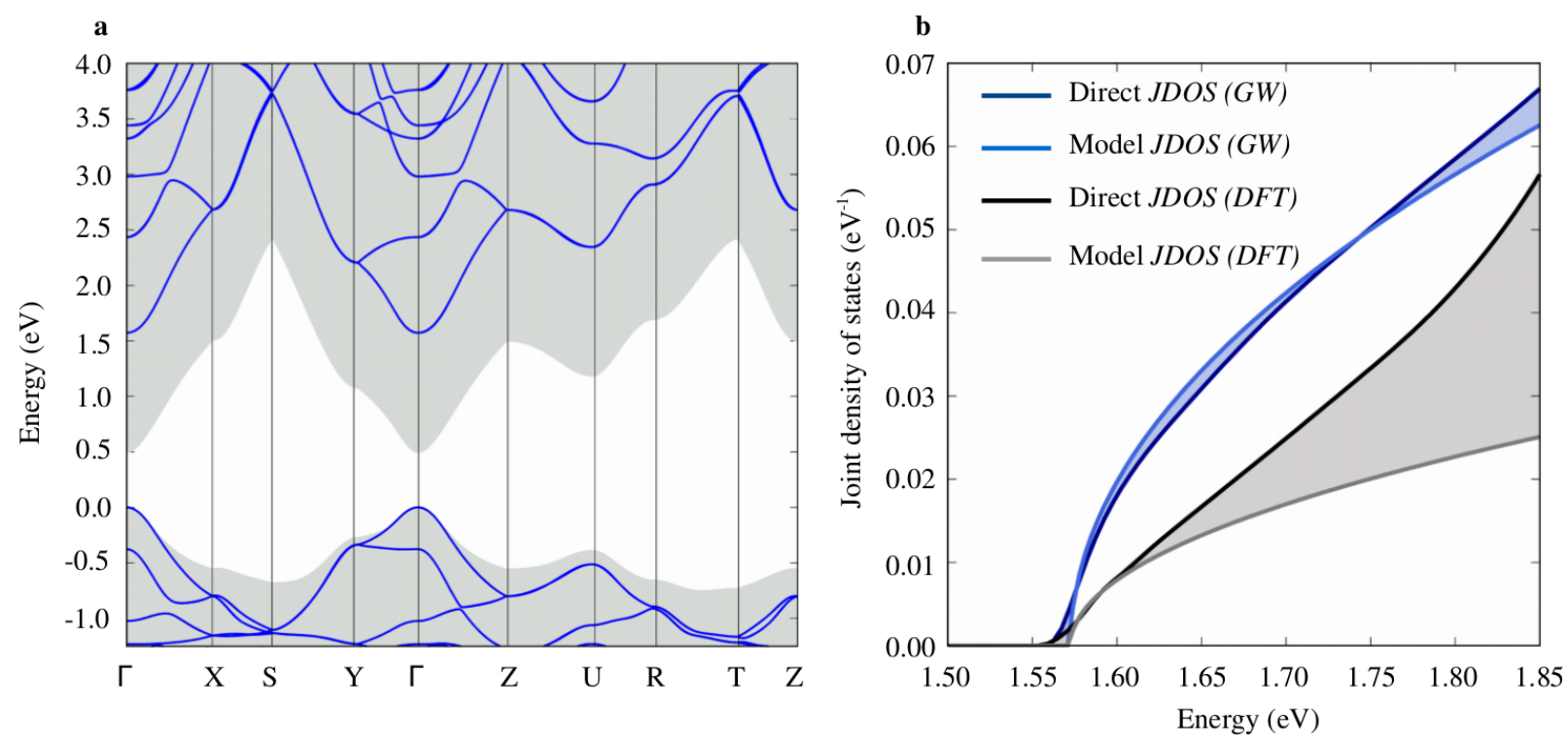

FIG. 4 Band parabolicity. a. Quasiparticle band structure of $\mathrm{CH}_{3} \mathrm{NH}_{3} \mathrm{PbI}_{3}$. The quasiparticle band structure is represented by the blue lines, the grey background is the DFT/LDA band structure. The quasiparticle band structure was obtained by performing a Wannier interpolation of the quasiparticle eigenvalues calculated for a $4 \times 4 \times 4 \Gamma$-centered grid, using the $G W$ and the self-consistent scissor scheme (Davies et al.|| 2017|, Filip et al., |2015). b. Joint density of states of $\mathrm{CH}_{3} \mathrm{NH}_{3} \mathrm{PbI}_{3}$ calculated within DFT (black line) and $G W$ (dark blue line). The direct joint densities of states were calculated using the definition $J(\omega)=\sum_{c v \mathbf{k}} \delta\left(\epsilon_{c \mathbf{k}}-\epsilon_{v \mathbf{k}}-\omega\right)$, where $c$ and $v$ are the band indices for the conduction and valence band states respectively, and $\mathbf{k}$ is the crystal momentum. The summation over the crystal momenta is performed by discretizing the Brillouin zone on a $\Gamma$ centered grid of $40 \times 40 \times 40$ points. The DFT and $G W$ eigenvalues are obtained on this grid from Wannier interpolation (Davies et al.||2017||Filip et al.|, 2015). The model joint densities of states (light blue line for $G W$ and grey for DFT) are calculated using the assumption that the bands are parabolic using, $J^{\mathrm{DFT} / G W}(\omega)=1 / 4 \pi^{2}\left(2 \mu^{\mathrm{DFT} / G W} / \hbar^{2}\right)^{3 / 2}\left(\omega-E_{g}\right)^{1 / 2}$, where $\mu$ is the reduced effective mass calculated from DFT $\left(0.06 m_{\mathrm{e}}\right)$ or $G W\left(0.11 \mathrm{~m}_{\mathrm{e}}\right)$. Both the model and the direct JDOS calculated from DFT were rigidly shifted in order to match the $G W$ onset, for clarity.

et al. 2015) describes the precise contribution of these three categories of vibrations to each of the vibrational modes. These contributions were calculated by a systematic decomposition of each mode followed by a factorgroup analysis of their symmetries.

Using the calculated vibrational frequencies of $\mathrm{CH}_{3} \mathrm{NH}_{3} \mathrm{PbI}_{3}$, the IR spectrum is obtained by calculating the absorption spectrum in the IR frequency range as (Pérez-Osorio et al., 2015):

$$
I(\omega)=\frac{e^{2}}{M_{0}} \sum_{\alpha \nu}\left|Z_{\alpha \nu}^{*}\right|^{2} \delta\left(\omega-\omega_{\nu}\right),
$$

where $M_{0}$ is the average mass over the unit cell, $e$ is the electronic charge, $\omega_{\nu}$ are the vibrational eigenfrequencies at the zone center and $Z_{\alpha \nu}^{*}=\sum_{\kappa \beta} \sqrt{\frac{M_{0}}{M_{\kappa}}} e_{\kappa \beta, \nu} Z_{\kappa, \alpha \beta}^{*}$, with $M_{\kappa}$ being the nuclear mass of atom $\kappa$ in the unit cell, $e_{\kappa \beta, \nu}$ the vibrational eigenmode of atom $\kappa$ in the Cartesian direction $\beta$, with vibrational frequency $\omega_{\nu}$, and $Z_{\kappa, \alpha \beta}^{*}$ is the Born effective charge (Pérez-Osorio et al. 2015 .

In FIG. 5 we show a comparison between the experimental and theoretical IR spectra of $\mathrm{CH}_{3} \mathrm{NH}_{3} \mathrm{PbI}_{3}$, as mea- sured and calculated by (Pérez-Osorio et al. 2015). Each of the features in the experimental IR spectrum is assigned to a peak and a vibrational symmetry in the calculated spectrum. The theoretical and experimental spectra agree well for almost the entire frequency range. The two main discrepancies are shown in the FIG.. 55-f. At high frequency, the three peaks are redshifted by approximately $70 \mathrm{~cm}^{-1}$. This discrepancy is shown by (PérezOsorio et al. 2015) to be corrected by the use of the generalized gradient approximation (GGA) instead of LDA for the calculation of the exchange-correlation potential in DFPT. In the low frequency (FIG. 55-d) region of the spectrum, DFPT calculations appear to overestimate the vibrational frequencies of the librational modes of $\mathrm{CH}_{3} \mathrm{NH}_{3}^{+}$around $150 \mathrm{~cm}^{-1}$. This discrepancy (FIG. 5pf) is consistent with other studies in literature (Družbicki et al. 2016, Mosconi et al., 2014), and it appears regardless of the choice of exchange-correlation functional (LDA vs. GGA), inclusion of relativistic spin-orbit coupling effects, van der Waals interactions or anharmonic effects (Pérez-Osorio et al. 2017). Moreover, (PérezOsorio et al. 2017) show that the calculated vibrational density of states is in good agreement with inelastic neutron scattering spectra (Družbicki et al., 2016), suggesting that the discrepancy between the experimental and 


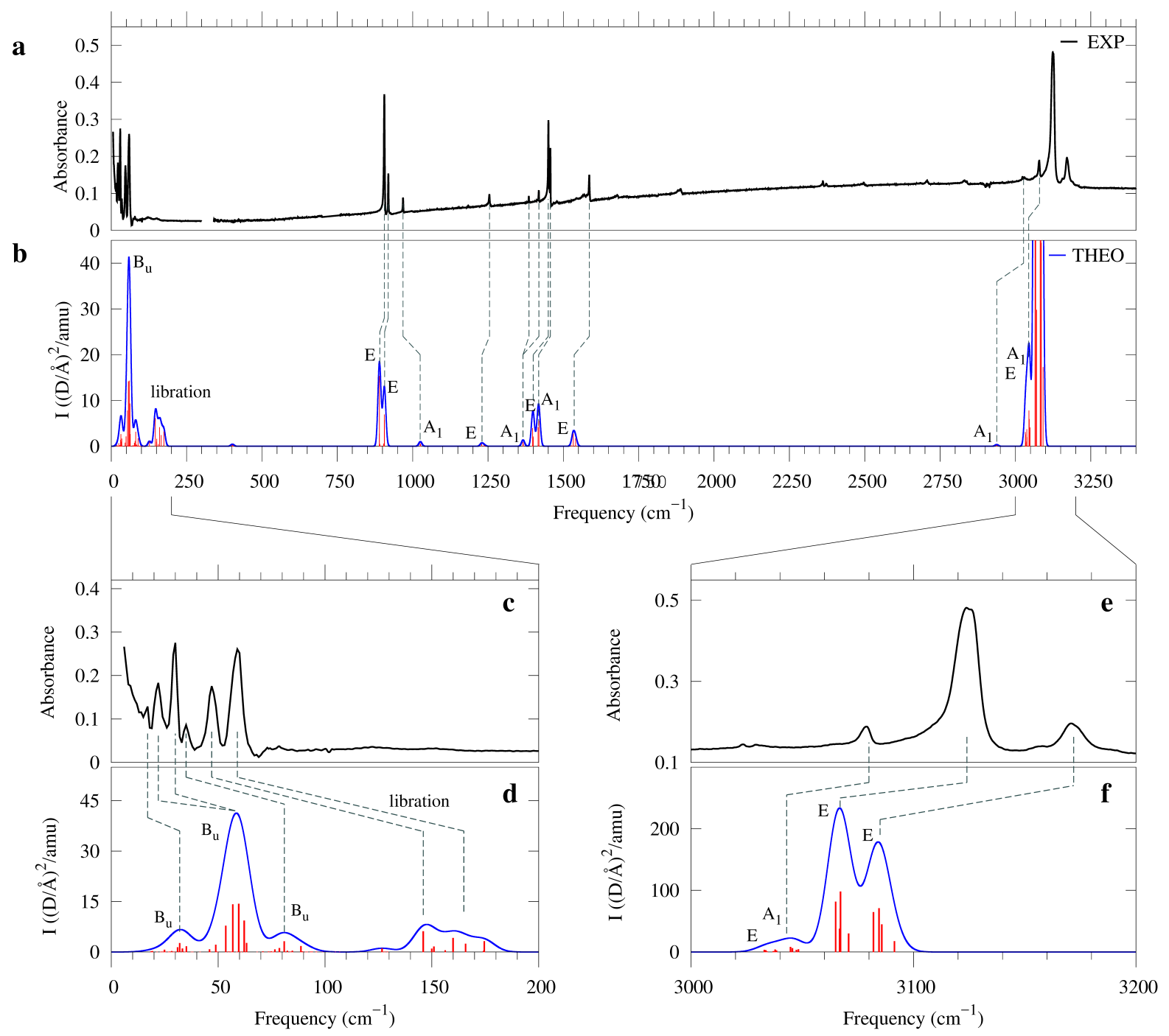

FIG. 5 Experimental and theoretical IR spectra of $\mathbf{C H}_{3} \mathbf{N H}_{3} \mathbf{P b I}_{3}$ at low temperature. a. IR spectrum of $\mathrm{CH}_{3} \mathrm{NH}_{3}$ measured on thin film at $10 \mathrm{~K}$. The dashed lines indicate the assignment of the most prominent peaks of the spectrum to features in the calculated IR spectrum in $\mathbf{b}$. The IR spectrum is measured at normal incidence, and only TO normal modes can be excited in this geometry. b. Calculated IR spectrum of $\mathrm{CH}_{3} \mathrm{NH}_{3} \mathrm{PbI}_{3}$ in the low temperature orthorhombic structure. The red sticks are the calculated infra-red intensities and the blue line corresponds to the spectrum calculated by representing each absorption peak with a Gaussian broadening. To be able to compare with the experimental data obtained within the geometry described in a, only TO modes are included in the calculated IR spectrum, as discussed by (Pérez-Osorio et al. 2015). c, d. Close-up of the comparison between the experimental (c) and theoretical (d) IR spectra in the low frequency region. e, f. Close-up of the comparison between experimental (e) and theoretical (f) IR spectra in the high-frequency region. Reprinted with permission from (Pérez-Osorio et al. 2015), Copyright (2015) American Chemical Society.

calculated IR spectra may be related to a mismatch between the experimental and theoretical IR intensities.

Having established both the electronic and vibrational properties of $\mathrm{CH}_{3} \mathrm{NH}_{3} \mathrm{PbI}_{3}$, we have access to the high and low frequency dielectric permitivities, $\epsilon_{\infty}$ and $\epsilon_{0}$, respectively. The high-frequency permitivity does not depend on lattice vibrations, but its value does depend on the calculated band gap. For this reason, the closest agreement with the experimental value $\varepsilon_{\infty}=6.5(\mathrm{Hi}-$ rasawa et al. , 1994) is obtained from finite-electric field calculations within scalar-relativistic DFT (Filip et al. 2015, Pérez-Osorio et al., 2015) (5.8, see Table I). The low-frequency permitivity depends on the vibrational frequencies of $\mathrm{CH}_{3} \mathrm{NH}_{3} \mathrm{PbI}_{3}$, with the vibrations of the inorganic $\mathrm{PbI}_{6}$ network carrying the largest contribution (Pérez-Osorio et al., 2015). (Pérez-Osorio et al. 2015) reports a value of $\varepsilon_{0}=25.3$, as calculated from DFPT, in very good agreement with the experimental low-frequency dielectric constant $\varepsilon_{0}=30.5$ (Poglitsch and Weber, 1987). As shown by (Pérez-Osorio et al. 
2015), the large difference between the high- and lowfrequency dielectric permitivities is due almost entirely to the vibrations of the inorganic $\mathrm{PbI}_{6}$ network.

\section{Electron-Phonon Coupling in $\mathbf{C H}_{3} \mathbf{N H}_{3} \mathbf{P b l}_{3}$}

Understanding the interaction between electrons and lattice vibrations is essential in the investigation of the transport properties and charge carrier recombinations in $\mathrm{CH}_{3} \mathrm{NH}_{3} \mathrm{PbI}_{3}$. In particular, electron-phonon coupling manifests itself prominently in the temperature dependent charge carrier mobility and in the broadening of the photoluminescence spectra (Johnston and Herz, 2016). The charge carrier mobility decreases with increasing temperature as $T^{m}$, where $m$ takes values between -1.4 and -1.6 (Brenner et al., 2015, Wright et al., 2016). Such behaviour is typical of non-polar semiconductors such as silicon or germanium, where electron-phonon interactions at room temperature are almost exclusively governed by the interaction with acoustic phonons. It is, however, unexpected in the case of $\mathrm{CH}_{3} \mathrm{NH}_{3} \mathrm{PbI}_{3}$, given the polar nature of the this compound.

As shown by (Wright et al. 2016), the nature of electronphonon interactions in $\mathrm{CH}_{3} \mathrm{NH}_{3} \mathrm{PbI}_{3}$ can be investigated by analyzing the photoluminescence linewidth as a function of temperature. In their study, (Wright et al., 2016) find that the contribution of acoustic phonons to the broadening of the photoluminescence linewidth is negligible compared to that of the longitudinal-optical (LO) modes. This argument is further corroborated by first principles calculations of the electron-phonon coupling, shown in FIG. 6. (Wright et al., 2016).

The photoluminescence linewidth can be directly associated with the imaginary part of the electron-phonon self energy, calculated using the following expression (Wright et al. 2016):

$$
\Sigma_{n \mathbf{k}}=\sum_{m \nu \mathbf{q}}\left|g_{m n}^{\nu}(\mathbf{k}, \mathbf{q})\right|^{2}\left[\frac{n_{\mathbf{q}, \nu}+f_{m \mathbf{k}+\mathbf{q}}}{\varepsilon_{n \mathbf{k}}-\varepsilon_{m \mathbf{k}+\mathbf{q}}-\omega_{\mathbf{q} \nu}-i \eta}+\frac{n_{\mathbf{q}, \nu}+1-f_{m \mathbf{k}+\mathbf{q}}}{\varepsilon_{n \mathbf{k}}-\varepsilon_{m \mathbf{k}+\mathbf{q}}-\omega_{\mathbf{q} \nu}-i \eta}\right],
$$

where $f_{m \mathbf{k}+\mathbf{q}}$ and $n_{\mathbf{q}, \nu}$ are the Fermi-Dirac and BoseEinstein occupations, $\epsilon_{n \mathbf{k}}$ and $\hbar \omega_{\mathbf{q} \nu}$ are the electron and phonon energies respectively, $\eta$ is a small broadening parameter, and $g_{m n}^{\nu}(\mathbf{k}, \mathbf{q})$ is the electron-phonon matrix element calculated by taking into account only the interaction with the LO phonons, as discussed by (Verdi and Giustino, 2015).

FIG. 6a shows how the imaginary part of the electron and hole linewidth are distributed across the electronic states in the quasiparticle band structure (Wright et al., 2016). The linewidth increases as the density of states increases (FIG.6p), because more states become available for electronic transitions. The predominant contribution to the electron-phonon self-energy comes from the LO phonon mode with an energy of $13 \mathrm{meV}$, which corresponds to the vibrations shown in FIG.6r. This value is in excellent agreement with the dominant phonon energy of $11 \mathrm{meV}$ determined from the experiments (Wright et al. 2016 ). FIG. 6d shows that the measured and the calculated trend for the PL broadening as a function of temperature are in very good agreement, thereby confirming that the predominant electron-phonon interaction is that between charge carriers and longitudinal optical phonons. In addition, both theoretical and experimental results reported by (Wright et al. 2016) show that $\mathrm{CH}_{3} \mathrm{NH}_{3} \mathrm{PbBr}_{3}$ exhibits an electron-phonon coupling which is $40 \%$ stronger than for the iodide, pointing to potential directions for controlling such effects through chemical substitution.

\section{E. Desirable electronic structure properties and the basis for materials design}

Experimental and theoretical studies of the electronic and optical properties of $\mathrm{CH}_{3} \mathrm{NH}_{3} \mathrm{PbI}_{3}$ have so far elucidated many of its fundamental properties. Perhaps the most striking finding is that $\mathrm{CH}_{3} \mathrm{NH}_{3} \mathrm{PbI}_{3}$ exhibits optoelectronic properties which are remarkably similar to the best inorganic semiconductors such as GaAs. Despite much progress on the understanding of the fundamental properties of $\mathrm{CH}_{3} \mathrm{NH}_{3} \mathrm{PbI}_{3}$ and related compounds, several important questions remain to be addressed. For example it would be important to investigate charge carrier transport and recombination processes (Herz, 2016), degradation mechanisms (Leijtens et al. 2017 ), and defect physics (Ball and Petrozza, 2017). All these topics are active areas of current research in this field.

Much of the current success of halide perovskites owes to the continued in-depth investigation of their fundamental optoelectronic properties, but also to the development of practical strategies to control these properties through chemical substitution and to design new materials with targeted functionalities. For example, chemical substitution has been systematically explored as a means to tune the optical absorption properties (Eperon et al. 2016, Filip et al. 2014), improve the stability (McMeekin et al. 2016) and reduce the toxicity (Giustino and Snaith, 2016) of lead-halide perovskites.

In particular, there is currently an ongoing search for alternative lead-free halide perovskites which retain the 
a

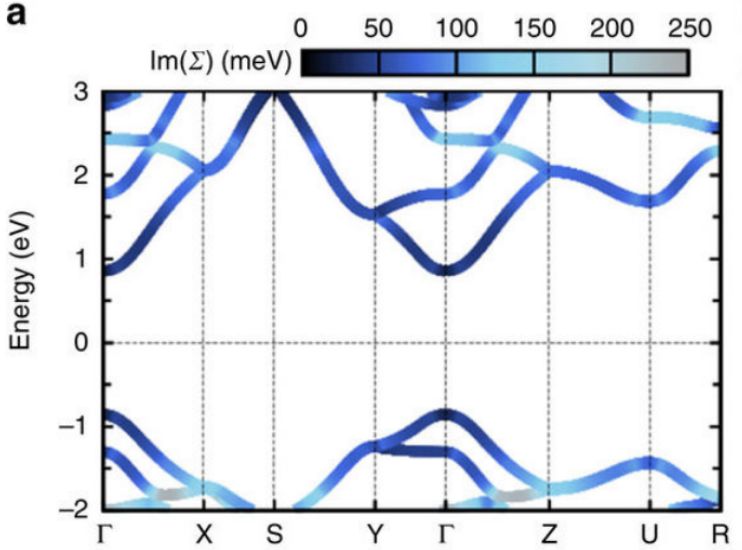

b

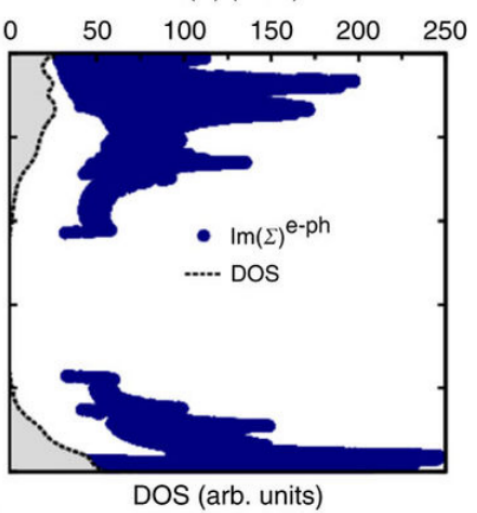

C

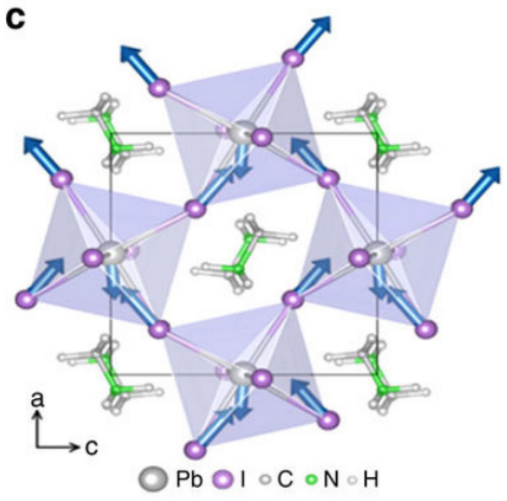

d

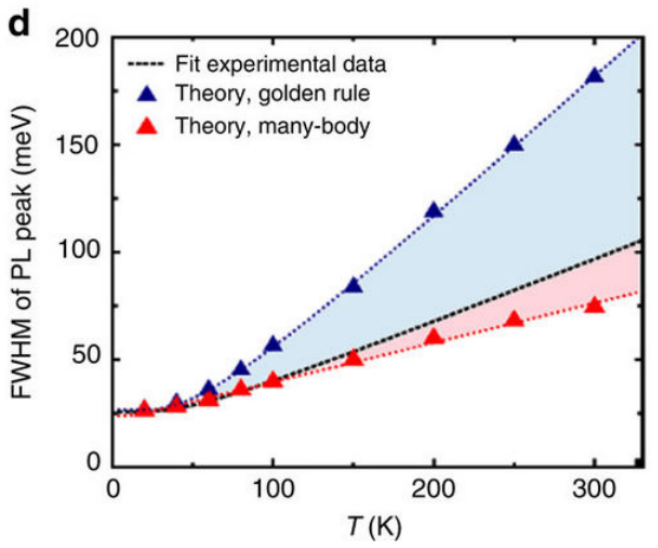

FIG. 6 Electron-phonon coupling and PL broadening of $\mathbf{C H}_{3} \mathbf{N H}_{3} \mathbf{P b} \mathbf{I}_{3}$. a. $G W$ quasiparticle band structure of the orthorhombic $\mathrm{CH}_{3} \mathrm{NH}_{3} \mathrm{PbI}_{3}$, combined with the heat map of the imaginary part of the electron-phonon self-energy, $\operatorname{Im}(\Sigma)$ at $\mathrm{T}$ $=200 \mathrm{~K}$. The zero of the energy is placed in the middle of the band gap. $\mathbf{b}$. Imaginary part of the electron-phonon self-energy (dark blue line) and the electronic density of states (dotted black line). $2 \operatorname{Im}(\Sigma)$ corresponds to the electron-hole linewidth arising from electron-phonon coupling (without the quasiparticle renormalization factor $Z$ ). c. Ball-and-stick representation of the LO vibration responsible for the broadening of the PL peaks. d. Temperature dependence of the full width at half maximum (FWHM) of the PL peak in $\mathrm{CH}_{3} \mathrm{NH}_{3} \mathrm{PbI}_{3}$. The dashed black line corresponds to the fit to experimental data and the blue and red triangles correspond to theoretical calculations using Fermi's golden rule and the Brillouin Wigner perturbation theory (red triangles), respectively. The theoretical broadening is obtained as the sum of $2 \operatorname{Im}(\Sigma)$ at the valence and conduction band edges, rigidly shifted by a constant inhomogeneous broadening of $26 \mathrm{meV}$. Reprinted with permission from (Wright et al. 2016), Copyright (2016) Nature Publishing Group.

optoelectronic properties of $\mathrm{CH}_{3} \mathrm{NH}_{3} \mathrm{PbI}_{3}$, and remove any potential environmental concerns due to the presence of lead. The nearly ideal optoelectronic properties of lead-halide perovskites set a very high bar for potential lead-free candidates: new compounds must exhibit a band gap in the visible range, long carrier lifetimes, good charge carrier mobilities, low exciton binding energies, and shallow or electrically-inactive defects. In the next section we review our efforts in the search for novel halide perovskites that might exhibit this unique combination of optoelectronic properties.

\section{DESIGN OF LEAD-FREE PEROVSKITES}

The presence of lead in lead-halide perovskites has raised questions on the environmental impact of perovskite solar cells. While the content of lead by weight in these devices is well below the limits set by governmental agencies, the prospect of developing lead-free perovskites is very attractive. Until now a lead-free material that can compete with $\mathrm{CH}_{3} \mathrm{NH}_{3} \mathrm{PbI}_{3}$ and related compounds has not been found, but substantial progress has been made in the development of new lead-free perovskites.

Among the different approaches to develop new leadfree perovskites, first-principles computational design has emerged as a powerful tool for screening new materials and identifying promising candidates. In fact, over the 
last two years several new lead-free halide double perovskites have been first proposed by theoretical studies, and subsequently synthesized in the lab. In the next sections representative examples in this area are discussed.

\section{A. Homovalent $\mathrm{Pb}$ replacement}

In order to replace lead in the $\mathrm{APbX}_{3}$ structure, the simplest starting point is to search for alternative cations in the +2 oxidation state. Hypothetical new perovskites should be stable towards air, moisture, illumination, and heat, and should exhibit band gap and carrier effective masses similar to those of $\mathrm{CH}_{3} \mathrm{NH}_{3} \mathrm{PbI}_{3}$. The most obvious choice would be to replace $\mathrm{Pb}^{2+}$ with another atom of Group IV, such as $\mathrm{Sn}^{2+}$. Indeed Sn-based perovskites were shown to exhibit ideal band gaps for photovoltaics applications, as well as good charge carrier mobilities (Stoumpos et al. 2013). However, perovskites containing $\mathrm{Sn}^{2+}$ tend do degrade rapidly due to the oxidation of the cation to $\mathrm{Sn}^{4+}$. This limitation was recently overcome by developing mixed $\mathrm{Sn} / \mathrm{Pb}$ halide perovskites, and the first applications to tandem device architectures are very promising (Eperon et al., 2016, McMeekin et al., 2016). A comprehensive search for other +2 B-site cations was performed by (Filip and Giustino, 2016). In this work the Periodic Table was screened by means of DFT calculations, using the procedure illustrated in FIG. 7. The candidate cations include metals which exhibit a stable +2 oxidation state and which form $\mathrm{BX}_{2}$ salts with $\mathrm{X}$ $=\mathrm{Cl}$, Br, or I. This choice leads to 116 hypothetical compounds for which the electronic structure was calculated using DFT/LDA, using the same orthorhombic perovskite structure as for $\mathrm{CH}_{3} \mathrm{NH}_{3} \mathrm{PbI}_{3}$. The calculated band structures were then used to reduce the materials space, and only those insulating compounds with a band gap smaller than $3.5 \mathrm{eV}$ were retained, leaving 40 potential candidates. These compounds were tested for dynamical stability using a 'shake-and-relax' approach, meaning that the structures are randomly distorted and subsequently re-optimised. Only structures that preserve the perovskite connectivity are retained in this step. For the 32 compounds that pass this test, more refined calculations including spin-orbit coupling effects were performed, and 25 hypothetical perovskites with band gaps smaller than $2 \mathrm{eV}$ were identified. For the compounds with the most promising electronic properties (Filip and Giustino, 2016) performed additional calculations at the $G W$ level. However in all the cases considered the quasiparticle band gaps were too wide for solar cell applications. This result suggests that homovalent $\mathrm{Pb}$ replacement may not be the best option for eliminating $\mathrm{Pb}$ in halide perovskites. A possible promising exception is the use of $\mathrm{Sn}^{2+}$, provided the stability issues can be resolved. Similar conclusions were reached by an extensive, highthroughput search of novel $\mathrm{ABX}_{3}$ perovskites by (Korbel et al. 2016).

\section{B. Heterovalent $\mathrm{Pb}$ replacement}

In the previous section we considered the possibility of replacing $\mathrm{Pb}$ with another divalent cation. In order to broaden the search space several authors considered the potential replacement of $\mathrm{Pb}^{2+}$ by cations in their +3 or +4 formal oxidation states. This choice leads to the formation of perovskite-related structures such as for example $\mathrm{Cs}_{3} \mathrm{Sb}(\mathrm{III})_{2} \mathrm{I}_{9}$ and $\mathrm{Cs}_{3} \mathrm{Bi}_{2} \mathrm{I}_{9}$ (Park et al., 2015; Saparov et al., 2015), $\mathrm{Cs}_{2} \mathrm{Sn}(\mathrm{IV}) \mathrm{I}_{6}$, and $\mathrm{Cs}_{2} \mathrm{PdBr}_{6}$ (Lee et al. | 2014, Sakai et al. 2017). In all these structures the three-dimensional corner-sharing connectivity of the octahedra is disrupted. In particular, $\mathrm{A}_{2} \mathrm{BX}_{6}$ compounds can be described as vacancy-ordered double perovskites, where the B-sites of the perovskite lattice are replaced alternatively by the +4 cation and by a vacancy. In this structure the $\mathrm{BX}_{6}$ octahedra are structurally disconnected. Similarly $\mathrm{A}_{3} \mathrm{~B}_{2} \mathrm{X}_{9}$ compounds can be thought of as if obtained from the perovskite lattice by removing one layer of octahedra. In both cases the resulting compounds behave electronically as low-dimensional systems (zero-dimensional and two-dimensional, respectively), and as a consequence the optoelectronic properties are not ideal (Xiao et al., 2017b).

Another potential strategy for lead replacement is the split-cation approach. This consists of replacing pairs of $\mathrm{Pb}^{2+}$ cations by pairs of +1 and +3 cations, so as to preserve the average oxidation state on the B-site. When the +1 and +3 cations are arranged in a rock-salt sublattice the structure is an ordered double perovskite, also known as elpasolite, as shown in FIG. 8. In this case the three-dimensional connectivity of the octahedra is maintained.

Double perovskites are well known in the literature on oxide perovskites (Vasala and Karppinen, 2015). Halide double perovskites are less known, but are commonly employed in radiation detectors as scintillators Biswas and Du, 2012, van Loef et al., 2002). Common elpasolites exhibit wide band gaps, therefore they are unsuitable for photovoltaics applications. In the following three sections we review recent findings on three new classes of halide elpasolites: halide double perovskites based on pnictogens and noble metals (Filip et al., 2016, McClure et al., 2016, Slavney et al., 2016, Volonakis et al., 2016); indium-silver halide double perovskites (Volonakis et al., 2017a; Zhao et al. 2017a); and indium-bismuth double perovskites (Volonakis et al., 2017b; Xiao et al., 2017a; Zhao et al., 2017b). 


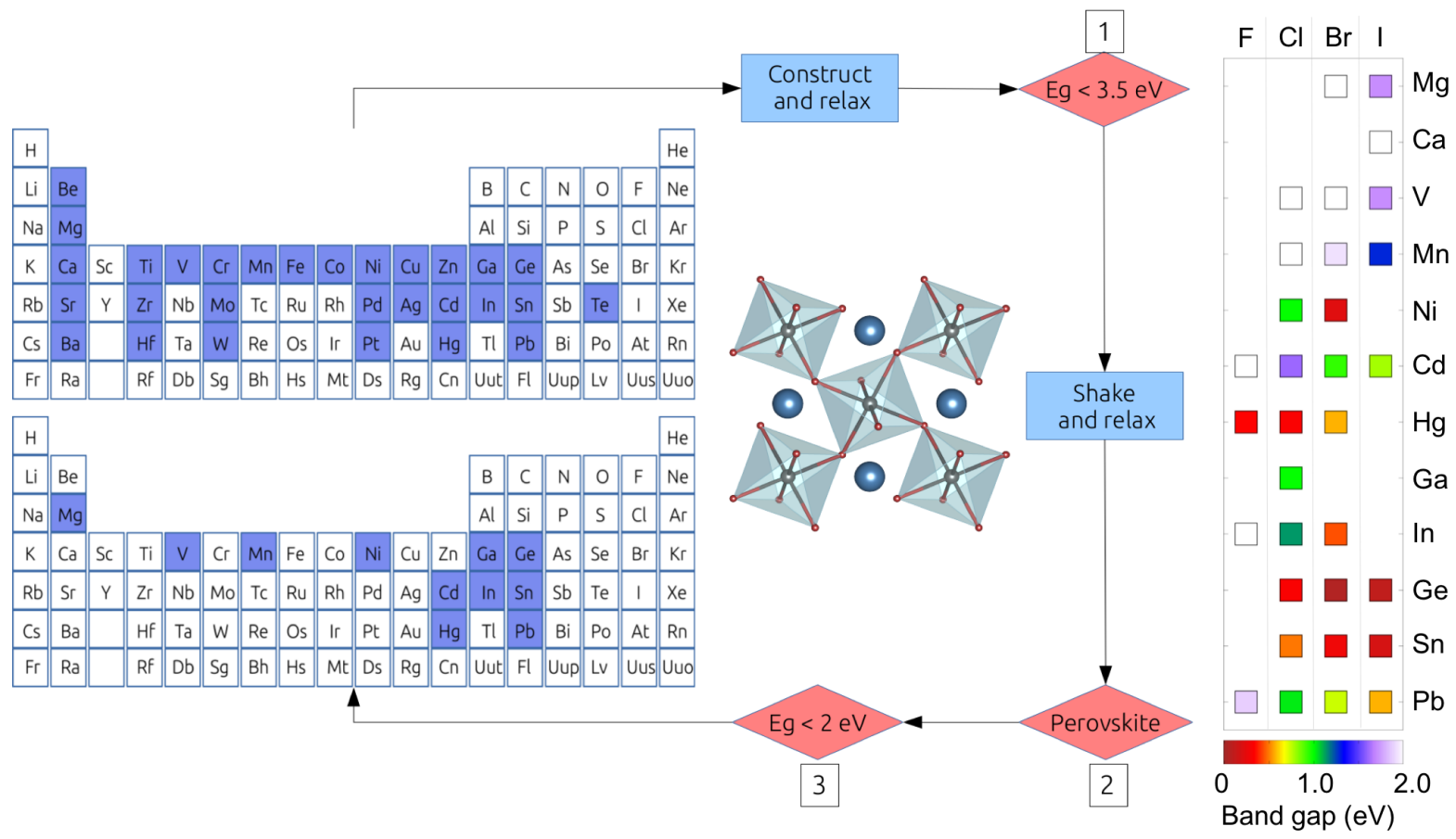

FIG. 7 Homovalent replacement of lead. Schematic illustration of the methodology and the selection criteria (in red triangles) employed for screening the Periodic Table for stable lead-free $\mathrm{ABX}_{3}$ halide perovskites. At first, all perovskite structures with a DFT/LDA band gap larger than $3.5 \mathrm{eV}$ were eliminated. The remaining compounds were randomly distorted ('shake-and-relax') to probe their dynamical stability and only compounds which retained the perovskite structure and exhibited a band gap of less than $2.0 \mathrm{eV}$ were considered for the final $G W$ calculations. These compounds are marked with the blue squares in the lower Periodic Table. The DFT/LDA band gaps for all the halide perovskites which satisfied all selection criteria are summarised at the left side of the figure. Adapted with permission from (Filip and Giustino, 2016), Copyright (2016) American Chemical Society.
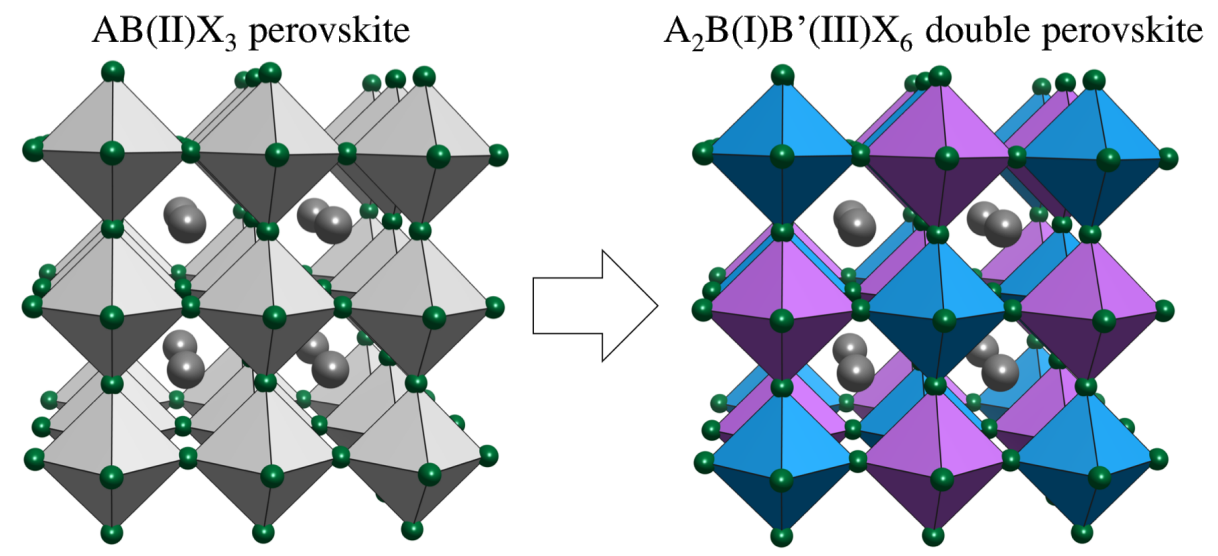

FIG. 8 The double perovskite structure. Two divalent cations at the B-site of the AB(II) $\mathrm{X}_{3}$ perovskite structure (left), are replaced with a monovalent and a trivalent cation to form the double perovskite $\mathrm{A}_{2} \mathrm{~B}(\mathrm{I}) \mathrm{B}^{\prime}$ (III) $\mathrm{X}_{6}$ structure (right). The two-types of octahedra in the double perovskite remain corner-sharing. When the B(I) and B'(III) cations are ordered in a rock-salt sublattice these compounds are called elpasolites (Meyer, 1980, Meyer and Gaebell, 1978, Morss et al., 1970).

\section{Double perovskites based on pnictogens and noble metals}

In order to design new lead-free halide double perovskites (Volonakis et al. . 2016) started from the known elpasolite $\mathrm{Cs}_{2} \mathrm{NaBiCl}_{6}$ (Morss and Robinson, 1972), and considered the replacement of monovalent $\mathrm{Na}$ by a noble metal. The rationale behind this strategy is that noble metals are known to be good electrical conductors due to their $s^{1}$ electrons. (Volonakis et al. 2016) performed DFT calculations for hypothetical compounds $\mathrm{Cs}_{2} \mathrm{NmPnX}_{6}$, where 
a
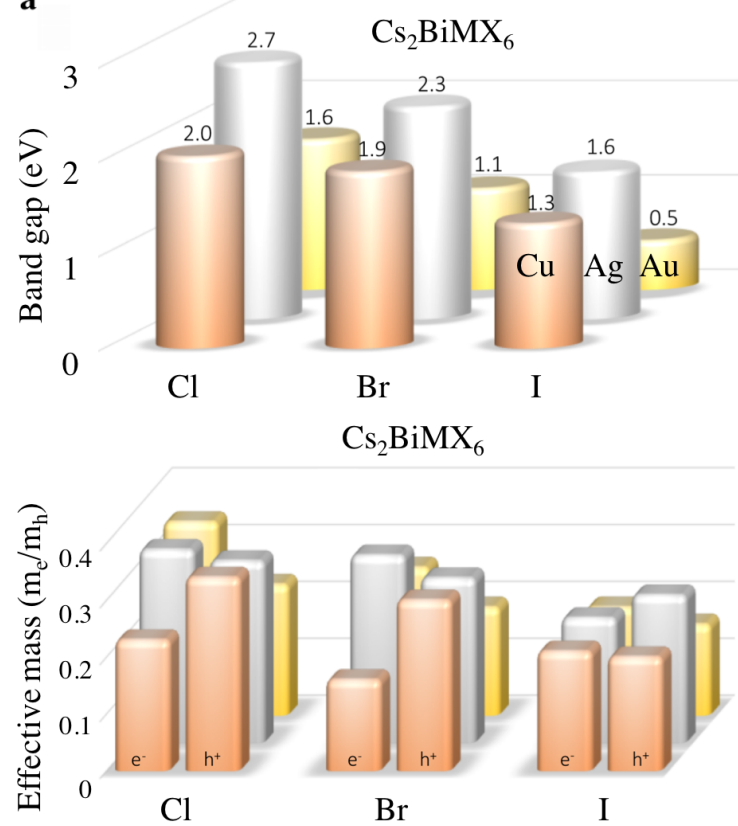

b
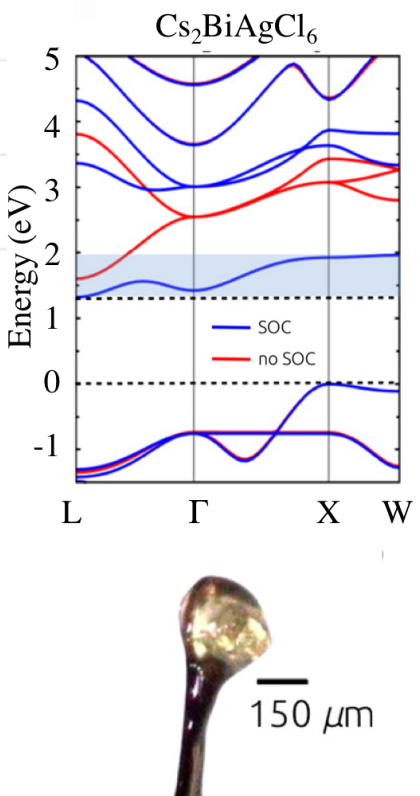
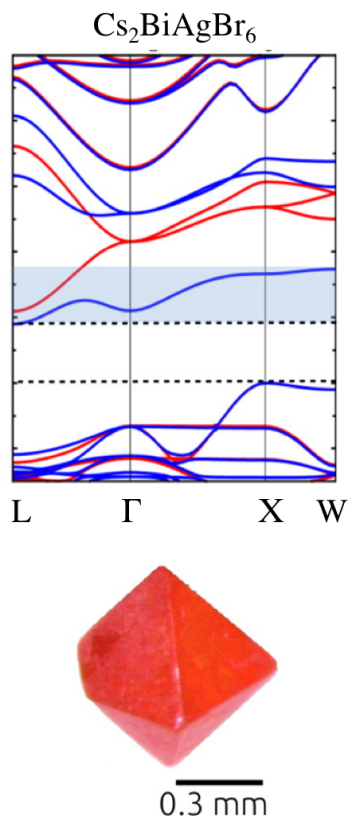

FIG. 9 Electronic properties of the pnictogen/noble metal double perovskites. a. Calculated electronic band gaps (top left) and effective masses (bottom left) of all the hypothetical $\mathrm{Cs}_{2} \mathrm{BiB}^{\prime} \mathrm{X}_{6}$ lead-free halide double perovskites, with $\mathrm{B}^{\prime}=$ $\mathrm{Cu}, \mathrm{Ag}, \mathrm{Au}$ and $\mathrm{X}=\mathrm{Cl}, \mathrm{Br}, \mathrm{I}$. All the band gaps are calculated by employing the PBE0 hybrid functional, and are found to be indirect and below $2.7 \mathrm{eV}$. The effective masses are low $\left(<0.4 \mathrm{~m}_{e}\right)$, as calculated from the DFT/LDA at the valence band top and the conduction band bottom for the holes and electrons, respectively. $\mathbf{b}$. The DFT/LDA electronic band structure for the synthesized $\mathrm{Cs}_{2} \mathrm{AgBiCl}_{6}$ and $\mathrm{Cs}_{2} \mathrm{AgBiBr}_{6}$ compounds. Calculations with (without) spin-orbit coupling are shown in blue (red), and the shaded area highlights the lowest unoccupied band. The synthesized $\mathrm{Cs}_{2} \mathrm{AgBiCl}_{6}$ and $\mathrm{Cs}_{2} \mathrm{AgBiBr}_{6}$ single-crystals $_{3}$ are shown below. Adapted with permission from (Volonakis et al. , 2016) and (Filip et al., 2016), Copyright (2016) American Chemical Society.

$\mathrm{Nm}=\mathrm{Cu}, \mathrm{Ag}, \mathrm{Au}, \mathrm{Pn}=\mathrm{Bi}, \mathrm{Sb}$, and $\mathrm{X}=\mathrm{Cl}, \mathrm{Br}, \mathrm{I}$. The calculated band gaps and carrier effective masses for the Bi-based hypothetical perovskites are shown in FIG. 9 a. All band gaps fall below $2.7 \mathrm{eV}$, and the effective masses do not exceed $0.4 m_{e}$. These results indicate that pnictogen/noble metal halide double perovskites might indeed be promising for optoelectronic applications. (Volonakis et al. 2016$)$ reported the synthesis of $\mathrm{Cs}_{2} \mathrm{AgBiCl}_{6}$. The compound was synthesized by following the same route as for $\mathrm{Cs}_{2} \mathrm{NaBiCl}_{6}$ (Morss and Robinson, 1972), and replacing $\mathrm{AgCl}$ for $\mathrm{NaCl}$. The structure of this compound was characterized by powder and single crystal X-ray diffraction (XRD) measurements. The authors obtained an ordered double perovskite structure with the $F m \overline{3} m$ face centered cubic space-group (no. 255) at room temperature. The reported lattice constant is $10.78 \AA$, in good agreement with the predicted DFT/LDA value of $10.50 \AA$. Following the same synthetic route (Filip et al. 2016) reported the new double perovskites $\mathrm{Cs}_{2} \mathrm{AgBiBr}_{6}$. Also in this case the XRD measurements confirmed the formation of an elpasolite structure, with a lattice constant of $11.26 \AA$. This value is slightly larger than for $\mathrm{Cs}_{2} \mathrm{AgBiCl}_{6}$, as expected based on the ionic radii of the halides. The synthesis of these compounds via solution and via solid-state synthesis was also reported indepen- dently (and a few weeks earlier) by two other groups (McClure et al., 2016, Slavney et al., 2016).

The electronic band structures of the new double perovskites are shown in FIG. 9b. In both cases the band gap is indirect, with the valence band top at the $X$ point of the Brillouin zone, and the conduction band bottom at the $L$ point. The band gaps calculated using the PBE0 hybrid functional are $2.7 \mathrm{eV}$ and $2.3 \mathrm{eV}$ for $\mathrm{Cs}_{2} \mathrm{AgBiCl}_{6}$ and $\mathrm{Cs}_{2} \mathrm{AgBiBr}_{6}$, respectively (Volonakis et al. , 2016). Quasiparticle $G W$ calculations yield slightly smaller gaps, namely $2.4 \mathrm{eV}$ and $1.8 \mathrm{eV}$, respectively (Filip et al., 2016$)$. The calculated band gaps are broadly in agreement with the measured optical gaps, which range between 2.2-2.8 eV and 1.9-2.2 eV for $\mathrm{Cs}_{2} \mathrm{AgBiCl}_{6}$ and $\mathrm{Cs}_{2} \mathrm{AgBiBr}_{6}$, respectively (Filip et al. 2016).

More recently (Filip et al. 2018) performed a systematic DFT investigation of the thermodynamic stability of the entire family of $\mathrm{Cs}_{2} \mathrm{NmPnX}_{6}$ compounds. The calculations involve the comparison between the total energy of each perovskite and the total energies of all possible decomposition products. This study predicted that only three double perovskites should be stable, namely $\mathrm{Cs}_{2} \mathrm{AgBiCl}_{6}, \mathrm{Cs}_{2} \mathrm{AgBiBr}_{6}, \mathrm{Cs}_{2} \mathrm{AgSbCl}_{6}$, in line with experimental observations (McClure et al., 2016; Slavney 
et al. 2016, Tran et al., 2017; Volonakis et al., 2016).

Among these new compounds, the double perovskite $\mathrm{Cs}_{2} \mathrm{AgBiBr}_{6}$ attracted considerable interest as a potential new material for photovoltaics and optoelectronic applications. In fact, solar cells using $\mathrm{Cs}_{2} \mathrm{AgBiBr}_{6}$ as the active layer were demonstrated by (Greul et al., 2017 ), and power conversion efficiencies of $2.5 \%$ were reported. Furthermore, the possibility of mixing $\mathrm{Cs}_{2} \mathrm{AgBiBr}_{6}$ with $\mathrm{CH}_{3} \mathrm{NH}_{3} \mathrm{PbI}_{3}$ in order to reduce the $\mathrm{Pb}$ content of perovskite solar cells (as opposed to completely replace $\mathrm{Pb}$ ) is also under investigation (Du et al. $2017 \mathrm{a}$ b). Finally, (Pan et al. 2017 ) showed that single crystals of $\mathrm{Cs}_{2} \mathrm{AgBiBr}_{6}$ can be employed to realize X-ray detectors with low detection thresholds (Pan et al. 2017).

\section{Double perovskites based on $\ln (\mathrm{III})$ and $\mathrm{Ag}(\mathrm{I})$}

Following up on the successful design and synthesis of lead-free halide double perovskites based on pnictogen/noble metals combinations, several groups set to improve the design by targeting double perovskites with a direct band gap. (Volonakis et al. 2017a) reasoned that the origin of the indirect band gap of $\mathrm{Cs}_{2} \mathrm{AgBiBr}_{6}$ lies in the hybridization between $\mathrm{Bi} s$-states and $\mathrm{Ag} d$-states near the valence band top. In order to demonstrate this effect, the authors artificially lowered the energy of the $\mathrm{Bi} s$-states in $\mathrm{Cs}_{2} \mathrm{AgBiBr}_{6}$ using an effective Hubbard potential in the DFT calculations. This strategy led to the appearance of a direct band gap at the zone center, in line with expectations. In order to exploit this finding, (Volonakis et al. 2017a) proposed to replace Bi with a +3 cation with the outermost $s$-shell unoccupied. This proposal, combined with the observation that the synthetic route to prepare $\mathrm{Cs}_{2} \mathrm{InNaCl}_{6}$ via solution was already known (Meyer and Gaebell, 1978, Morss and Robinson, 1972), motivated the consideration of hypothetical compounds of the type $\mathrm{Cs}_{2} \mathrm{InAgX}_{6}$ with $\mathrm{X}=\mathrm{Cl}$ or $\mathrm{Br}$ (Volonakis et al., 2017a).

The calculated band structure of $\mathrm{Cs}_{2} \mathrm{InAgCl}_{6}$ is shown in FIG. 10a. As expected the system exhibits a direct gap at the $\Gamma$ point, and the top of the valence band is comprised of $\mathrm{Ag}$ and In $d$-orbitals, hybridized with the halogen $p$ orbitals. The band gap of $\mathrm{Cs}_{2} \mathrm{InAgCl}_{6}$ was predicted to be in the range $2.7 \pm 0.6 \mathrm{eV}$, with the error bar reflecting the spread in band gaps obtained from calculations based on different exchange and correlation functionals. This compound was successfully synthesized via an acidic solution synthesis route by mixing stoichiometric amounts of $\mathrm{InCl}_{3}, \mathrm{AgCl}$ and $\mathrm{CsCl}$. Powder XRD measurements confirmed the formation of a double perovskite with a face-centered cubic crystal ( $F m \overline{3} m$ space group) and a lattice constant of $10.47 \AA$, in good agreement with the DFT predictions (Volonakis et al., 2017a).

The nature of $\mathrm{In} / \mathrm{Ag}$ cation ordering in $\mathrm{Cs}_{2} \mathrm{InAgCl}_{6}$ is not firmly established yet. The characteristic fingerprints of cation ordering in the XRD spectra of double perovskites are two doublets arising from reflections at the planes containing each cation (FIG. 10b). In contrast to the cases of $\mathrm{Cs}_{2} \mathrm{AgBiCl}_{6}$ and $\mathrm{Cs}_{2} \mathrm{AgBiBr}_{6}$, where such doublets have clearly been identified (Volonakis et al., 2016), the assignment proved more difficult for $\mathrm{Cs}_{2} \mathrm{In} \mathrm{AgCl}_{6}$. (Volonakis et al., 2017a) calculated the XRD patterns for structures with ordered or disordered cations and compared these results to measured spectra, as shown in FIG. 10p. From this comparison it is clear that the difference between the two structures is too subtle to be resolved by the measurements. More refined XRD data from (Zhou et al. . 2017), taken on single crystal samples, show a clear doublet at low angle. This supports the notion that $\mathrm{Cs}_{2} \mathrm{InAgCl}_{6}$ is a fully-ordered elpasolite, although further investigations would be desirable to settle this question.

The calculated electron and hole effective masses of $\mathrm{Cs}_{2} \mathrm{InAgCl}_{6}$ are relatively light, $0.29 m_{e}$ and $0.28 m_{e}$, respectively. These values refer to the parabolic bands that can be seen in FIG. 10a. The non-dispersive band that is seen along the $\Gamma X$ direction in the same figure arises from two-dimensional wavefunctions comprising of In $4 \mathrm{~d}_{x^{2}-y^{2}}$ states and $\mathrm{Cl} 3 p_{x, y}$-states. These states are expected to hinder hole transport along the six equivalent [001] directions, as well as to favor the formation of deep traps.

The nature of the band gap of $\mathrm{Cs}_{2} \mathrm{InAgCl}_{6}$ remains an open question. The absorption data reported by (Volonakis et al. 2017a) indicate an absorption onset around $3.3 \mathrm{eV}$, which is consistent with the upper range obtained from DFT calculations. However the photoluminescence data by (Volonakis et al. $2017 \mathrm{a}$ ) also indicate emission near $2.0 \mathrm{eV}$, indicating the presence of optically-active defects within the gap. (Luo et al., 2018) proposed an alternative explanation for these effects, involving parityforbidden band-to-band transitions.

While the band gap of $\mathrm{Cs}_{2} \mathrm{InAgCl}_{6}$ is too wide for applications in photovoltaics, this new double perovskites is attracting attention for applications in UV detectors due to its excellent stability and non-toxicity. In particular (Luo et al., 2018) and (Zhou et al., 2017) succeeded in growing mm-sized single crystals, and (Luo et al., 2018) fabricated UV detectors with high on/off ratios, fast photoresponse, low dark current, and high sensitivity.

\section{Double perovskites based on $\mathrm{Bi}(\mathrm{III})$ and $\ln (\mathrm{I})$}

The new double perovskites described in the preceding sections either exhibit indirect band gaps, or nondispersive bands at the band extrema. In order to move a step closer to realizing high-quality semiconducting halide perovskites without lead, (Volonakis et al. 2017b) revisited the general design principles of the split-cation approach based on +1 and +3 cations. 


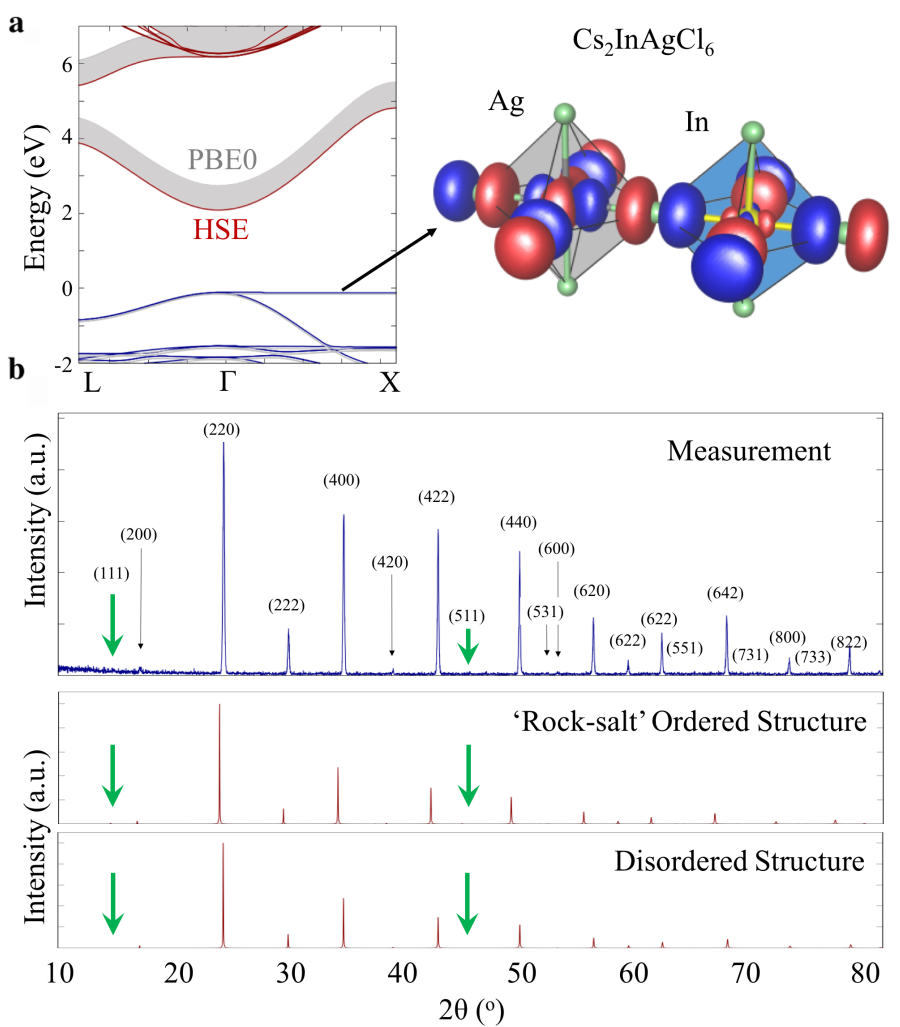

FIG. 10 Electronic and structural properties of $\mathbf{C s}_{2} \mathbf{I n} \mathbf{A g C l}_{6}$. a. Electronic band structure of $\mathrm{Cs}_{2} \mathrm{InAgCl}_{6} \mathrm{within}_{\mathrm{n}}$ the DFT/PBE0 and the DFT/HSE hybrid functionals. Square moduli of the electronic wavefunctions for the highest (top) and second highest (bottom) occupied state along the $\Gamma$-X direction. b. Measured powder XRD pattern of $\mathrm{Cs}_{2} \mathrm{InAgCl}_{6}$ (top), XRD pattern calculated by assuming ideal rock-salt ordering of the In and Ag cations (middle), and XRD pattern calculated by assuming a fully disordered cation sublattice (bottom). The green arrows show the fingerprints of the cation ordering. The difference between the calculated ordered and disordered patterns are within the noise level of the measured spectrum, therefore it is not possible to unambiguously confirm cation ordering in this case. Adapted with permission from (Volonakis et al. 2017a), Copyright (2017) American Chemical Society.

The authors argued that an ideal double perovskite should contain cations with the same valence configuration as $\mathrm{Pb}^{2+}$. This implies that candidate elements should have (i) occupied $s$-orbitals, (ii) unoccupied $p$ orbitals, and (iii) a filled $d$ shell far from the band edge. The candidates for the monovalent cation are limited to the alkali metals, the noble metals and the Group III elements (boron group). Among these options only the latter have occupied $s$-orbitals and unoccupied $p$-orbitals in their formal +1 oxidation state. However, only two members of this group, In and $\mathrm{Tl}$, are stable as +1 cations, and $\mathrm{Tl}$ is to be excluded due to its toxicity. This leaves $\mathrm{In}^{+}$as the sole candidate for the monovalent Bsite cation. For the trivalent B-site cation the choice is between pnictogens, some transition metals such as Sc, $\mathrm{Y}, \mathrm{Cu}$ and $\mathrm{Au}$ that were previously reported to form elpasolites (Giustino and Snaith, 2016), elements of the boron group in the +3 oxidation state, lanthanides and actinides. Among these elements only the pnictogens satisfy the criteria (i)-(iii) above. In this group $\mathrm{N}$ and $\mathrm{P}$ are too small to coordinate six halogens in a octahedral arrangement, and As is toxic, therefore the only options left are $\mathrm{Sb}^{3+}$ and $\mathrm{Bi}^{3+}$. Based on this simple reasoning (Volonakis et al. , 2017b) concluded that the best option for replacing $\mathrm{Pb}$, from the electronic structure viewpoint, is to consider $\operatorname{In}(\mathrm{I}) / \mathrm{Sb}$ (III) and $\operatorname{In}(\mathrm{I}) / \mathrm{Bi}(\mathrm{III})$ double perovskites.

Building on this insight, (Volonakis et al. $2017 \mathrm{~b}$ ) evaluated the stability of the hypothetical compounds $\mathrm{A}_{2} \mathrm{InPnX}_{6}$ with $\mathrm{A}=\mathrm{K}, \mathrm{Rb}, \mathrm{Cs}, \mathrm{Pn}=\mathrm{Sb}, \mathrm{Bi}$, and $\mathrm{X}=\mathrm{F}$, $\mathrm{Cl}$, Br, I, using both the Goldschmidt tolerance analysis and DFT calculations of decomposition energies in conjunction with the Materials Project Database (Jain et al. 2013). The outcome of this study was that all compounds are unstable with respect to decomposition, in line with the work of (Xiao et al. $2017 \mathrm{a})$. However $\mathrm{Cs}_{2} \mathrm{InBiBr}_{6}$ was found to be only marginally unstable, as the calculated decomposition energy of $1 \mathrm{meV}$ per formula unit falls within the uncertainty of the computational method. This finding raises the hope that $\mathrm{Cs}_{2} \mathrm{InBiBr}_{6}$ or a closely related compound might be amenable to synthesis. (Volonakis et al. 2017b) also noted that the decomposition energy correlates with the size of the A-site cation, and that double perovskites with larger A-site 
a

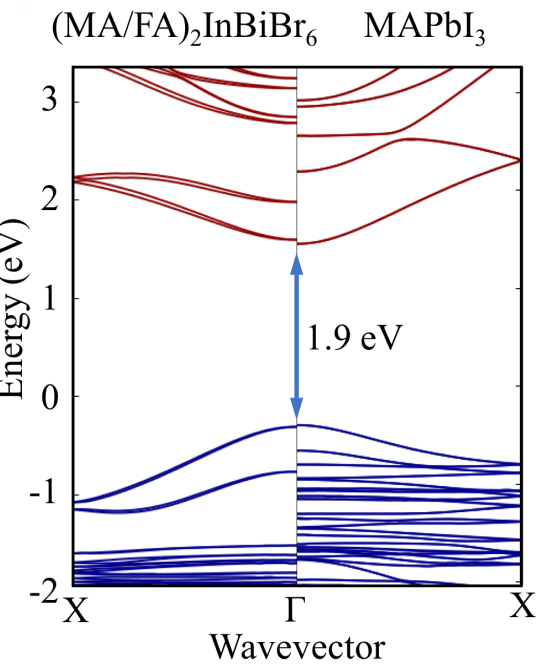

b

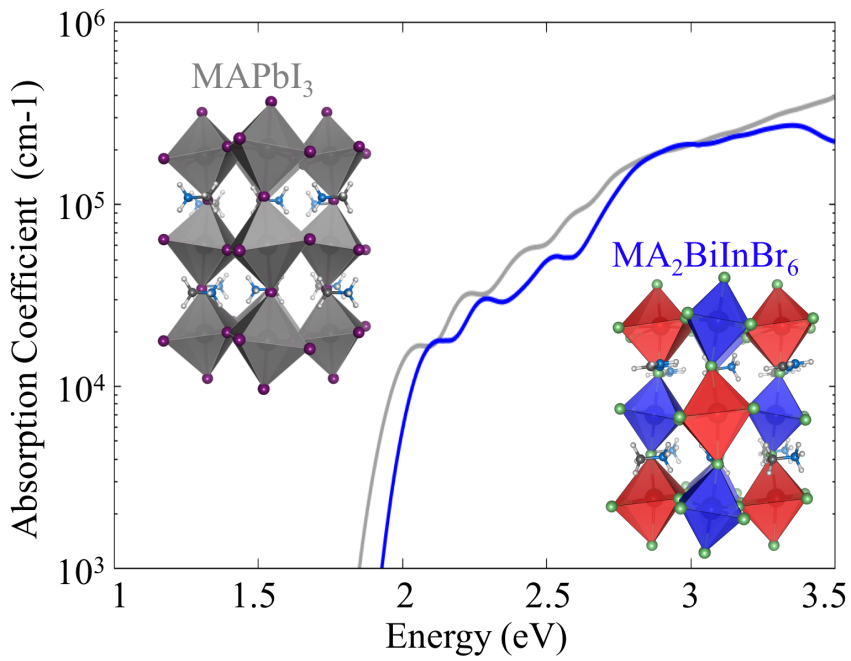

FIG. 11 Indium/Bismuth halide double perovskites. a. Comparison between the electronic band structures of the hypothetical lead-free double perovskite $(\mathrm{MA} / \mathrm{FA})_{2} \mathrm{InBiBr}_{6}$ and the lead halide perovskite $\mathrm{MAPbI}_{3}$. MA stands for methylammonium $\left(\mathrm{CH}_{3} \mathrm{NH}_{3}\right)$, and $\mathrm{FA}$ for formamidinium $\left[\mathrm{CH}\left(\mathrm{NH}_{2}\right)_{2}\right]$. Calculations were performed by employing the PBE0 hybrid functional. The double perovskite structures were fully optimized starting from the low-temperature $P$ nma phase of $\mathrm{MAPbI}_{3}$, and by replacing two $\mathrm{Pb}$ atoms with $\mathrm{In}$ and $\mathrm{Bi}$. b. Calculated absorption coefficients for $\mathrm{MA}_{2} \mathrm{InBiBr}_{6}\left(\mathrm{blue}\right.$ line), and $\mathrm{MAPbI} \mathrm{I}_{3}$ (grey line). Adapted with permission from (Volonakis et al., 2017b), Copyright (2017) American Chemical Society.

cations tend to be less prone to decomposition. Based on this observation the authors proposed that $\mathrm{In}(\mathrm{I}) / \mathrm{Bi}(\mathrm{III})$ halide double perovskites might be amenable for synthesis by using large organic cations such as $\mathrm{CH}_{3} \mathrm{NH}_{3}^{+}$and $\mathrm{CH}\left(\mathrm{NH}_{2}\right)_{2}^{+}$.

The electronic band structure of the hypothetical $\left(\mathrm{CH}_{3} \mathrm{NH}_{3} / \mathrm{CH}\left(\mathrm{NH}_{2}\right)_{2}\right)_{2} \mathrm{InBiBr}_{6}$ double perovskites along with the band structure of $\mathrm{CH}_{3} \mathrm{NH}_{3} \mathrm{PbI}_{3}$ are shown in FIG. 11a. As anticipated, owing to the similar electronic configuration of $\mathrm{Pb}^{2+}, \mathrm{In}^{+}$and $\mathrm{Bi}^{3+}$ the two compounds exhibit very similar band structures. The band gaps are direct in both cases and of the same magnitude. Similarly the calculated optical absorption spectra for these compounds are very similar, as shown in FIG. 11p. These findings highlight the potential of hybrid In/Bi halide double perovskites for photovoltaics and optoelectronics. At the time of writing this chapter synthetic attempts at making $\operatorname{In}(\mathrm{I}) / \mathrm{Bi}$ (III) halide double perovskites have not been successful. The key challenge appears to be the tendency of $\operatorname{In}(\mathrm{I})$ to be oxidized to the more stable form In(III), therefore future synthesis studies should devise strategies for maintaining In in its +1 state long enough to enable its incorporation in the double perovskite lattice.

\section{CONCLUSIONS}

In this chapter we have presented a detailed account of the contributions made by our materials modelling group to the study of the fundamental properties and design principles of metal-halide perovskites. The results reviewed in this chapter were obtained by employing techniques ranging from standard density functional theory and density functional perturbation theory to many-body perturbation theory treatments of electronic excitations and electron-phonon coupling phenomena. Our studies so far have been directed at two principal goals, understanding the fundamental properties of lead-halide perovskites and developing design principles to guide the discovery of novel lead-free metal-halide perovskite semiconductors.

The current understanding of the fundamental properties of lead-halide perovskites is due to a complementary effort combining state-of-the art predictive computational modelling and experimental measurements which have allowed us to rationalize the optoelectronic properties of these materials from the perspective of atomistic modelling. In addition, we have shown that the predictive power of computational modelling can be directed at designing new lead-free halide perovskites, which led most notably to the computational discovery of a new family of lead-free halide double perovskites. During the last year more than eight novel lead-free double perovskite compounds have been successfully synthesized (Deng et al., 2016; Filip et al. 2016; McClure et al., 2016, Slavney et al., 2016; Tran et al., 2017, Volonakis et al., 2016, 2017a; Wei et al., 2016, 2017). Among these, compounds such as $\mathrm{Cs}_{2} \mathrm{AgBiCl}{ }_{6}, \mathrm{Cs}_{2} \mathrm{AgBiBr}{ }_{6}, \mathrm{Cs}_{2} \mathrm{InAgCl}_{6}$ and $\mathrm{Cs}_{2} \mathrm{AgSbCl}_{6}$, were first proposed by us in silico.

Despite the tremendous popularity of halide perovskites, there are still fundamental open questions and tech- 
nological challenges that remain unresolved. A nonexhaustive list includes the fundamental understanding of charge transport and excitonic properties, elucidating the physics of defects and their impact on the degradation of these materials under standard solar cell operating conditions, and developing a practical solution for reducing the $\mathrm{Pb}$ content without impacting the device performance. All of these problems are currently active areas for both experimental and theoretical research.

In a broader context, the rapid rise of hybrid halide perovskites can be seen as a true success story for experimental and theoretical materials design. As the race for ever higher performing and cost-efficient optoelectronic devices continues, there is an increasing demand for the discovery of new functional materials. Thanks to the continued development of highly accurate $a b$ initio methods, computational materials modelling can now directly guide discovery, by designing materials and predicting their properties in silico ahead of synthesis. These developments place $a b$ initio modelling in a central role in modern materials science and engineering, as an accelerator for the discovery and deployment of new technologies.

\section{ACKNOWLEDGEMENT}

The research leading to these results has received funding from the Graphene Flagship (Horizon 2020 Grant No. 696656 - GrapheneCore1), the Leverhulme Trust (Grant RL-2012- 001), and the UK Engineering and Physical Sciences Research Council (Grant No. EP/J009857/1, EP/M020517/1 and EP/ L024667/1).

\section{REFERENCES}

Baikie, T, Y. Fang, J. M. Kadro, M. Schreyer, F. Wei, S. G. Mhaisalkar, M. Grätzel, and T. J. White (2013), "Synthesis and crystal chemistry of the hybrid perovskite $\left(\mathrm{CH}_{3} \mathrm{NH}_{3} \mathrm{PbI}_{3}\right)$ for solid-state sensitized solar applications." J. Chem. Mater. A 1, 5628.

Ball, J M, and A. Petrozza (2017), "Defects in perovskitehalides and their effects in solar cells," Nat. Energ. 1 (16149).

Biswas, Koushik, and Mao-Hua Du (2012), "Energy transport and scintillation of cerium-doped elpasolite $\mathrm{Cs}_{2} \mathrm{LiYCl}_{6}$ : Hybrid density functional calculations," Phys. Rev. B 86 (1), 014102-014109.

Brenner, T M, D. A. Egger, A. M. Rappe, L. Kronik, G. Hodes, and D. Cahen (2015), "Are mobilities in hybrid organic-inorganic halide perovskites actually high?" J. Phys. Chem. Lett. 6, 4754-4757.

Brivio, F, K. T. Bulter, A. Walsh, and M. van Schilfgaarde (2014), "Relativistic quasiparticle self-consistent electronic structure of hybrid halide perovskite photovoltaic absorbers," Phys. Rev. B 89, 155204.

Carignano, M A, and A. Kachmar (2015), "Thermal effects on $\mathrm{CH}_{3} \mathrm{NH}_{3} \mathrm{PbI}_{3}$ perovskite from ab initio molecular dy- namics simulations," J. Phys. Chem. C 119 (17), 89918997.

Chen, Y-S, J. S. Manser, and P. V. Kamat (2015), "All solution-processed lead halide perovskite- $\mathrm{BiVO}_{4}$ tandem assembly for photolytic solar fuels production," J. Am. Chem. Soc. 137 (2), 974-981.

Comin, R, G. Walters, E. S. Thibau, O. Voznyy, Z.-H. Lu, and E. H. Sargent (2015), "Structural, optical, and electronic studies of wide-bandgap lead halide perovskites," J. Mater. Chem. C 3, 8839-8843.

Davies, C L, M. R. Filip, J. B. Patel, T. W. Crothers, C. Verdi, A. D. Wright, R. L. Milot, F. Giustino, M. B. Johnston, and L. M. Herz (2017), "Bimolecular recombination in methylammonium lead triiodide perovskite: An inverse absorption process," Nat. Commun. 9, 293.

De Wolf, S, J. Holovsky, S.-J. Moon, P. Löper, B. Niesen, M. Ledinsky, F.-J. Haug, J.-H. Yum, and C. Ballif (2014), "Organometallic halide perovskites: Sharp optical absorption edge and its relation to photovoltaic performance," J. Phys. Chem. Lett. 5, 1035-1039.

Deng, Z, F. Wei, S. Sun, G. Kieslich, A. K. Cheetham, and P. D. Bristowe (2016), "Exploring the properties of lead-free hybrid double perovskites using a combined computational-experimental approach," J. Mater. Chem. A 4 (31), 12025-12029.

D'Innocenzo, V, G. Grancini, M. J. P. Alcocer, A. R. S Kandada, S. D. Stranks, M. M. Lee, G. Lanzani, Snaith H. J., and A. Petrozza (2014), "Excitons versus free charges in organo-lead tri-halide perovskites," Nat. Commun. 5, 3586.

Družbicki, K, R. S. Pinna, S. Rudić, M. Jura, G. Gorini, and F. Fernandez-Alonso (2016), "Unexpected cation dynamics in the low-temperature phase of methylammonium lead iodide: The need for improved models," J. Phys. Chem. Lett. 7 (22), 4701-4709.

Du, K-Z, W. Meng, X. Wang, Y. Yan, and D. B. Mitzi (2017a), "Bandgap engineering of lead-free double perovskite $\mathrm{Cs}_{2} \mathrm{AgBiBr}_{6}$ through trivalent metal alloying," Angew. Chem. Int. Ed. 56 (28), 8158-8162.

Du, K-Z, X. Wang, Q. Han, Y. Yan, and D. B. Mitzi (2017b), "Heterovalent B-site co-alloying approach for halide perovskite bandgap engineering," ACS Energ. Lett. 2, 24862490.

Elliott, R J (1957), "Intensity of the optical absorption by excitons," Phys. Rev. 108, 1384.

Eperon, G E, T. Leijtens, K. A. Bush, R. Prasanna, T. Green, J. T-W. Jacob Tse-Wei Wang, D. P. McMeekin, G. Volonakis, R. L. Milot, R. May, A. Palmstrom, D. J. Slotcavage, R. A. Belisle, J. B. Patel, E. S. Parrott, R. J. Sutton, W. Ma, F. Moghadam, B. Conings, A. Babayigit, H-G. Boyen, S. Bent, F. Giustino, L. M. Herz, M. B. Johnston, M. D. McGehee, and Snaith H. J. (2016), "Perovskite-perovskite tandem photovoltaics with optimized bandgaps," Science 354, 861-865.

Eperon, G E, S. D. Stranks, C. Menelaou, M. B. Johnston, L. M. Herz, and H. J. Snaith (2014), "Formamidinium lead trihalide: A broadly tunable perovskite for efficient planar heterojunction solar cells," Energ. Environ. Sci. 7, 982-988.

Even, J, L. Pedesseau, J.-M. Jancu, and C. Katan (2013), "Importance of spin-orbit coupling in hybrid organic/inorganic perovskites for photovoltaic applications," J. Phys. Chem. Lett. 119, 10161-10177.

Filip, M R, G. E. Eperon, H. J. Snaith, and F. Giustino (2014), "Steric engineering of metal-halide perovskites with tunable optical band gaps," Nat. Commun. 5, 5757. 
Filip, M R, and F. Giustino (2014), " $G W$ quasiparticle band gap of the hybrid organic-inorganic perovskite $\mathrm{CH}_{3} \mathrm{NH}_{3} \mathrm{PbI}_{3}$," Phys. Rev. B 90, 245145.

Filip, M R, and F. Giustino (2016), "Computational screening of homovalent lead substitution in organicinorganic halide perovskites," J. Phys. Chem. C 120, 166-173.

Filip, M R, S. Hillman, A. A. Haghighirad, H. J. Snaith, and F. Giustino (2016), "Band gaps of the lead-free halide double perovskites $\mathrm{Cs}_{2} \mathrm{BiAgCl}_{6}$ and $\mathrm{Cs}_{2} \mathrm{BiAgBr}_{6}$ from theory and experiment," J. Phys. Chem. Lett. 7, 2579-2585.

Filip, M R, X. Liu, A. Miglio, G. Hautier, and F. Giustino (2018), "Phase diagrams and stability of lead-free halide double perovskites $\mathrm{Cs}_{2} \mathrm{BB}^{\prime} \mathrm{X}_{6}$," J. Phys. Chem. C 122 (1), 158-170.

Filip, M R, C. Verdi, and F. Giustino (2015), " $G W$ band structure and carrier effective mases of $\mathrm{CH}_{3} \mathrm{NH}_{3} \mathrm{PbI}_{3}$ and hypothetical perovskites of the type $\mathrm{APbI}_{3}: \mathrm{A}=\mathrm{NH}_{4}$, $\mathrm{PH}_{4}, \mathrm{AsH}_{4}$ and $\mathrm{SbH}_{4}, "$ J. Phys. Chem. C 119, 2420924219.

Giustino, Feliciano, and Henry J. Snaith (2016), "Toward lead-free perovskite solar cells," ACS Energy Letters 1 (6), 1233-1240.

Greul, E, M. L. Petrus, A. Binek, P. Docampo, and T. Bein (2017), "Highly stable, phase pure $\mathrm{Cs}_{2} \mathrm{AgBiBr}_{6}$ double perovskite thin films for optoelectronic applications," J. Mater. Chem. A 5 (37), 19972-19981.

Hao, F, C. C. Stoumpos, R. P. H. Chang, and M. G. Kanatzidis (2014), "Anomalous band gap behavior in mixed sn and pb perovskites enables broadening of absorption spectrum in solar cells," J. Am . Chem. Soc. 136 (22), 8094-8099.

Hedin, L (1965), "New method for calculating the one-particle green's function with application to the electron-gas problem," Phys. Rev. 139, A796.

Herz, L M (2016), "Charge-carrier dynamics in organicinorganic metal halide perovskites," Annu. Rev. Phys. Chem. 67, 3.1-3.25.

Hirasawa, M, T. Ishihara, T. Goto, and N. Uchida, K.and Miura (1994), "Magnetoabsorption of the lowest exciton in perovskite-type compund $\left(\mathrm{CH}_{3} \mathrm{NH}_{3} \mathrm{PbI}_{3}\right)$," Phys. B 201, 427-430.

Hybertsen, M S, and S. G. Louie (1986), "Electron correlation in semiconductors and insulators: Band gaps and quasiparticle energies," Phys. Rev. B 34, 5390.

Jain, A, S. P. Ong, G. Hautier, W. Chen, W. D. Richards, S. Dacek, S. Cholia, D. Gunter, D. Skinner, G. Ceder, and K. A. Persson (2013), "The materials project: A materials genome approach to accelerating materials innovation," APL Mater. 1, 011002.

Johnston, M B, and L. M. Herz (2016), "Hybrid perovskites for photovoltaics: Charge-carrier recombination, diffusion, and radiative efficiencies," Acc. Chem. Res. 49 (1), 146154.

Kioupakis, E, P. Zhang, M. L. Cohen, and S. G. Louie (2008), " $G W$ quasiparticle corrections to the LDA+U/GGA+U electronic structure of bcc hydrogen," Phys. Rev. B 77, 155114

Korbel, S, M. A. L. Marques, and S. Botti (2016), "Stability and electronic properties of new inorganic perovskites from high-throughput ab initio calculations," J. Mater. Chem. C 4 (15), 3157-3167.

Lahnsteiner, J, G. Kresse, D. D. Kumar, A. Sarma, C. Franchini, and M. Bokdam (2016), "Room-temperature dynamic correlation between methylammonium molecules in lead-iodine based perovskites: An ab initio molecular dynamics perspective," Phys. Rev. B 94, 214114.

Ledinský, M, P. Löper, B. Niesen, J. Holovský, S.-J. Moon, J.-H. Yum, S. De Wolf, A. Fejfar, and C. Ballif (2015), "Raman spectroscopy of organic inorganic halide perovskites," J. Phys. Chem. Lett. 6, 401-406.

Lee, B, C. C. Stoumpos, N. Zhou, F. Hao, C. Malliakas, Yeh. C.-Y., T. J. Marks, M. G. Kanatzidis, and R. P. H. Chang (2014), "Air-stable molecular semiconducting iodosalts for solar cell applications: Cs2SnI6 as a hole conductor," J. Am. Chem. Soc. 136 (43), 15379-15385.

Lee, M M, J. Teuscher, T. Miyasaka, T. N. Murakami, and H. J. Snaith (2012), "Efficient hybrid solar cells based on meso-superstructured organometal halide perovskites," Science 338, 643-647.

Leijtens, T, K. Bush, R. Cheacharoen, R. Beal, A. Bowring, and M. D. McGehee (2017), "Towards enabling stable lead halide perovskite solar cells; interplay between structural, environmental, and thermal stability," J. Mater. Chem. A $\mathbf{5}, 11483$.

van Loef, E V D, P. Dorenbos, C. W. E. van Eijk, K. W. Krämer, and H. U. Güdel (2002), "Scintillation and spectroscopy of the pure and $\mathrm{Ce}^{3+}$-doped elpasolites: $\mathrm{Cs}_{2} \mathrm{LiYX}_{6}$ $(\mathrm{X}=\mathrm{Cl}, \mathrm{Br})$," Journal of Physics: Condensed Matter 14 (36), 8481.

Luo, J, S. Li, H. Wu, Y. Zhou, Y. Li, J. Liu, J. Li, K. Li, F. Yi, G. Niu, and J. Tang (2018), " $\mathrm{Cs}_{2} \mathrm{AgInCl}_{6}$ double perovskite single crystals: Parity forbidden transitions and their application for sensitive and fast UV photodetectors," ACS Photonics 5 (2), 398-405.

Marzari, N, and D. Vanderbilt (1997), "Maximally localized generalized Wannier functions for composite energy bands," Phys. Rev. B 56, 12847.

McClure, E T, M. R. Ball, W. Windl, and P. M. Woodward (2016), " $\mathrm{Cs}_{2} \mathrm{AgBiX}_{6}(\mathrm{X}=\mathrm{Br}, \mathrm{Cl})$ - new visible light absorbing, lead-free halide perovskite semiconductors," Chem. Mater. 28, 1348-1354.

McMeekin, D P, G Sadoughi, W. Rehman, G. E. Eperon, M. Saliba, M. T. Hörantner, A. A. Haghighirad, N. Sakai, L. Korte, B. Rech, M. B. Johnston, L. M. Herz, and H. J. Snaith (2016), "A mixed-cation lead mixed-halide perovskite absorber for tandem solar cells," Science 8, 151155 .

Megaw, H (1973), Crystal Structures. A working approach (W. B. Saunders Company, Philadelphia, London, Toronto).

Menéndez-Proupin, E, P. Palacios, P. Wahnón, and J. C. Conesa (2014), "Self-consistent relativistic band structure of the $\mathrm{CH}_{3} \mathrm{NH}_{3} \mathrm{PbI}_{3}$ perovskite," Phys. Rev. B 90, 045207.

Meyer, G (1980), "Halo-elpasolites. VI. the first iodoelpasolites, $\mathrm{Cs}_{2} \mathrm{~B}^{I} \mathrm{M}^{I I I} \mathrm{I}_{6}\left(\mathrm{~B}^{I}=\mathrm{Li}, \mathrm{Na}\right)$." Z. Naturforsch. B 35, 268-276.

Meyer, G, and H. Gaebell (1978), "Halo-elpasolites. iv. on bromo-elpasolites $\mathrm{Cs}_{2} \mathrm{~B}^{\mathrm{I}} \mathrm{M}^{\mathrm{III}} \mathrm{Br}_{6}, \mathrm{~B}^{\mathrm{I}}=\mathrm{Li}, \mathrm{Na} ; \mathrm{M}^{\mathrm{III}}=\mathrm{Sc}$, Y, La-Lu, In, V, Cr)," Z. Naturforsch. B 33 (12), 14761478.

Milot, R L, G. E. Eperon, H. J. Snaith, M. B. Johnston, and L. M. Herz (2015), "Temperature-dependent chargecarrier dynamics in $\mathrm{CH}_{3} \mathrm{NH}_{3} \mathrm{PbI}_{3}$ perovskite thin films," Adv. Func. Mater. 25, 6218-6227.

Miyata, A, A. Mitioglu, P. Plochocka, O. Portugall, J. T.-W. Wang, S. D. Stranks, H. J. Snaith, and R. J. Nicholas (2015), "Direct measurement of the exciton binding energy and effective masses for charge carriers in an organic- 
inorganic tri-halide perovskite," Nat. Phys. 11, 582-587.

Morss, L R, and W. R. Robinson (1972), "Crystal structure of $\mathrm{Cs}_{2} \mathrm{BiNaCl}_{6}$," Acta Cryst. B 28, 653-654.

Morss, L R, M. Siegal, L. Stenger, and N. Edelstein (1970), "Preparation of cubic chloro complex compounds of trivalent metals: $\mathrm{Cs}_{2}$ namcl $_{6}$," Inorg. Chem. 9 (7), 1771-1775.

Mosconi, E, C. Quarti, T. Ivanovska, G. Ruani, and De Angelis F. (2014), "Structural and electronic properties of organo-halide lead perovskites: a combined IRspectroscopy and ab initio molecular dynamics investigation," Phys. Chem. Chem. Phys. 16, 16137-16144.

Motta, C, F. El-Mellouhi, S. Kais, N. Tabet, F. Alharbi, and S. Sanvito (2015), "Revealing the role of organic cations in hybrid halide perovskite $\mathrm{CH}_{3} \mathrm{NH}_{3} \mathrm{PbI}_{3}$," Nat. Commun. 6 (7026).

Noh, J H, S. H. Im, J. H. Heo, T. N. Mandal, and S. I. Seok (2013), "Chemical management for colorful, efficient, and stable inorganicorganic hybrid nanostructured solar cells," Nano Lett. 13 (4), 1764-1769.

NREL, (2017), "National renewable energy laboratory: Best research-cell efficiencies," http://www.nrel.gov/ncpv/ images/efficiency_chart.jpg, accessed: Nov. 2017.

Pan, W, H. Wu, J. Luo, Z. Deng, C. Ge, C. Chen, X. Jiang, W.-J. Yin, G. Niu, L. Zhu, L. Yin, Y. Zhou, Q. Xie, X. Ke, M. Sui, and J. Tang (2017), " $\mathrm{Cs}_{2} \mathrm{AgBiBr}_{6}$ single-crystal X-ray detectors with a low detection limit," Nat. Photon. 11 (11), 726-732.

Park, B-W, B. Philippe, X. Zhang, H. Rensmo, G. Boschloo, and E. M. J. Johansson (2015), "Bismuth based hybrid perovskites $\mathrm{A}_{3} \mathrm{Bi}_{2} \mathrm{I}_{9}$ (A: Methylammonium or cesium) for solar cell application," Adv. Mater. 27 (43), 6806-6813.

Pérez-Osorio, M A, A. Champagne, M. Zacharias, G.-M. Rignanese, and F. Giustino (2017), "Van der waals interactions and anharmonicity in the lattice vibrations, dielectric constants, effective charges, and infrared spectra of the organic-inorganic halide perovskite $\mathrm{CH}_{3} \mathrm{NH}_{3} \mathrm{PbI}_{3}$," J. Phys. Chem. C 121, 18459-18471.

Pérez-Osorio, M A, R. L. Milot, M. R. Filip, J. B. Patel, L. M. Herz, M. B. Johnston, and F. Giustino (2015), "Vibrational properties of the organic-inorganic halide perovskite $\mathrm{CH}_{3} \mathrm{NH}_{3} \mathrm{PbI}_{3}$ from theory and experiment: Factor group analysis, first principles calaculations, and low-temperature infrared spectra," J. Phys. Chem. C 119, 25703-25718.

Poglitsch, A, and D Weber (1987), "Dynamic disorder in methylammoniumhalogenoplumbates (II) observed by millimeter-wave spectroscopy," J. Chem. Phys. 87, 6373.

van Roosbroek, W, and W. Shockley (1954), "Photonradiative recombination of electrons and holes in germanium," Phys. Rev. 94, 1558-1560.

Sakai, N, A. A. Haghighirad, M. R. Filip, P. K. Nayak, S. Nayak, A. Ramadan, Z. Wang, F. Giustino, and H. J. Snaith (2017), "Solution-processed cesium hexabromopalladate (IV), $\mathrm{Cs}_{2} \mathrm{PdBr}_{6}$, for optoelectronic applications," J. Am. Chem. Soc. 139 (17), 6030-6033.

Saliba, M, T. Matsui, K. Domanski, J.-Y. Seo, A. Ummadisingu, S. M. Zakeeruddin, J.-P. Correa-Baena, W. R. Tress, A. Abate, A. Hagfeldt, and M. Grätzel (2016), "Incorporation of rubidium cations into perovskite solar cells improves photovoltaic performance," Science 354 (6309).

Saparov, B, F. Hong, J.-P. Sun, H.-S. Duan, W. Meng, S. Cameron, I. G. Hill, Y. Yan, and D. B. Mitzi (2015), "Thin-film preparation and characterization of $\mathrm{Cs}_{3} \mathrm{Sb}_{2} \mathrm{I}_{9}$ : A lead-free layered perovskite semiconductor," Chem. Mater. 27 (16), 5622-5632.
Scherpelz, P, M. Govoni, I. Hamada, and G. Galli (2016), "Implementation and validation of fully relativistic $G W$ calculations: Spin-orbit coupling in molecules, nanocrystals, and solids," J. Chem. Thory Comput. 12 (8), 35233544 .

van Schilfegaarde, M, T. Kotani, and S. Faleev (2006), "Quasiparticle self-consistent $G W$ theory," Phys. Rev. Lett. 96, 226402.

Slavney, A H, T. Hu, A. M. Lindenberg, and H. I. Karunadasa (2016), "A bismuth-halide double perovskite with long carrier recombination lifetime for photovoltaic applications," J. Am. Chem. Soc. 138, 2138-2141.

Souza, I, N. Marzari, and D. Vanderbilt (2001), "Maximally localized Wannier functions for entangled energy bands," Phys. Rev. B 65, 035109.

Stoumpos, C C, C. D. Malliakas, and M. G. Kanatzidis (2013), "Semiconducting tin and lead iodide perovskites with organic cations: Phase transitions, high mobilities, and near-infrared photoluminescent properties," Inorganic Chemistry 52 (15), 9019-9038.

Stranks, S, and H. J. Snaith (2015), "Perovskites for photovoltaic and light-emitting devices," Nat. Nanotechnol. 10, 391-402.

Tan, Z-K, R.S. Moghaddam, M. L. Lai, P. Docampo, R. Higler, F. Deschler, M. Price, A. Sadhanala, L. M. Pazos, D. Credgington, F. Hanush, T. Bein, H. J. Snaith, and R. H. Friend (2014), "Bright light-emitting diodes based on organometal halide perovskites," Nat. Nanotechnol. 9, $687-692$.

Tran, T T, J. R. Panella, J. R. Chamorro, J. R. Morey, and T. M. McQueen (2017), "Designing indirect-direct bandgap transitions in double perovskites," Mater. Horiz. 4, 688693.

Vasala, S, and M. Karppinen (2015), " $\mathrm{A}_{2} \mathrm{~B}^{\prime} \mathrm{B}^{\prime} \mathrm{O}_{6}$ perovskites: A review," Prog. Solid State Chem. 43 (1), 1-36.

Verdi, C, and F. Giustino (2015), "Fröhlich electron-phonon vertex from first principles," Phys. Rev. Lett. 115 (17), 176401

Volonakis, G, M. R. Filip, A. A. Haghighirad, N. Sakai, B. Wenger, H. J. Snaith, and F. Giustino (2016), "Leadfree halide double perovskites via heterovalent substitution of noble metals," J. Phys. Chem. Lett. 7, 1254-1259.

Volonakis, G, A. A. Haghighirad, R. L. Milot, W. H. Sio, M. R. Filip, B. Wenger, M. B. Johnston, L. M. Herz, H. J. Snaith, and F. Giustino (2017a), " $\mathrm{Cs}_{2} \mathrm{InAgCl}_{6}$ : A new lead-free halide double perovskite with direct band gap," J Phys. Chem. Lett. 8 (4), 772-778.

Volonakis, G, A. A. Haghighirad, H. J. Snaith, and F. Giustino (2017b), "Route to stable lead-free double perovskites with the electronic structure of $\mathrm{CH}_{3} \mathrm{NH}_{3} \mathrm{PbI}_{3}$ : A case for mixed-cation $\left[\mathrm{Cs} / \mathrm{CH}_{3} \mathrm{NH}_{3} / \mathrm{CH}\left(\mathrm{NH}_{2}\right)_{2}\right]_{2} \mathrm{InBiBr}_{6}$," The Journal of Physical Chemistry Letters 8, 3917.

Ward, J W, H. L. Smith, A. Zeidell, P. J. Diemer, S. R. Baker, H. Lee, M. M. Payne, J. E. Anthony, M. Guthold, and O. D. Jurchescu (2017), "Solution-processed organic and halide perovskite transistors on hydrophobic surfaces," ACS Appl. Mater. Interf. 9 (21), 18120-18126.

Wehrenfennig, C, M. Liu, H. J. Snaith, M. B. Johnston, and L. M. Herz (2014), "Homogeneous emission line broadening in the organo lead halide perovskite $\mathrm{CH}_{3} \mathrm{NH}_{3} \mathrm{PbI}_{3 x} \mathrm{Cl}_{x}$," J. Phys. Chem. Lett. 5, 1300-1306.

Wei, F, Z. Deng, S. Sun, F. Xie, G. Kieslich, D. M. Evans, M. A. Carpenter, P. D. Bristowe, and A. K. Cheetham (2016), "The synthesis, structure and electronic properties 
of a lead-free hybrid inorganic-organic double perovskite $(\mathrm{MA})_{2} \mathrm{KBiCl}_{6}$ (MA = methylammonium)," Mater. Horiz. 3 (4), 328-332.

Wei, F, Z. Deng, S. Sun, F. Zhang, D. M. Evans, G. Kieslich, S. Tominaka, M. A. Carpenter, J. Zhang, P. D. Bristowe, and A. K. Cheetham (2017), "Synthesis and properties of a lead-free hybrid double perovskite: $\left(\mathrm{CH}_{3} \mathrm{NH}_{3}\right)_{2} \mathrm{AgBiBr}_{6}$," Chem. Mater. 29 (3), 1089-1094.

Weller, M T, O. J. Weber, P. F. Henry, A. M. DiPumpo, and T. C. Hansen (2015), "Complete structure and cation orientation in the perovskite photovoltaic methylammonium lead iodide between 100 and 352 K." Chem. Commun. 51, 4180-4183.

Wright, A D, C. Verdi, R. L. Milot, G. E. Eperon, M. A. Pérez-Osorio, H. J. Snaith, F. Giustino, M. B. Johnston, and L. M. Herz (2016), "Electron-phonon coupling in hybrid lead halide perovskites," Nat. Commun. 7 (11755).

Xiao, Z, K.-Z. Du, W. Meng, J. Wang, D. B. Mitzi, and
Y. Yan (2017a), "Intrinsic instability of $\mathrm{Cs}_{2} \operatorname{In}^{(I)} \mathrm{M}^{(I I I)} \mathrm{X}_{6}$ $(\mathrm{M}=\mathrm{Bi}, \mathrm{Sb} ; \mathrm{X}=$ halogen $)$ double perovskites: A combined density functional theory and experimental study," J. Am. Chem. Soc. 139 (17), 6054-6057.

Xiao, Z, W. Meng, J. Wang, D. B. Mitzi, and Y. Yan (2017b), "Searching for promising new perovskite-based photovoltaic absorbers: the importance of electronic dimensionality," Mater. Horiz. 4 (2), 206-216.

Zhao, X-G, D. Yang, Y. Sun, T. Li, L. Zhang, L. Yu, and A. Zunger (2017a), "Cu-In halide perovskite solar absorbers," J. Am. Chem. Soc. 139 (19), 6718-6725.

Zhao, X-G, J.-H. Yang, Y. Fu, D. Yang, Q. Xu, L. Yu, S.-H. Wei, and L. Zhang (2017b), "Design of lead-free inorganic halide perovskites for solar cells via cation-transmutation," J. Am. Chem. Soc. 139 (7), 2630-2638.

Zhou, J, Z. Xia, M. S. Molokeev, X. Zhang, D. Peng, and Q. Liu (2017), "Composition design, optical gap and stability investigations of lead-free halide double perovskite $\mathrm{Cs}_{2} \mathrm{AgInCl}_{6}$," J. Mater. Chem. A 5 (29), 15031-15037. 